\title{
Synaptic Organization of Anterior Olfactory Nucleus Inputs to Piriform Cortex
}

\author{
Marco J. Russo, ${ }^{1}$ Kevin M. Franks, ${ }^{1}$ Roxanne Oghaz, ${ }^{1}$ Richard Axel, ${ }^{2}$ and Steven A. Siegelbaum ${ }^{3}$ \\ ${ }^{1}$ Department of Neuroscience, Vagelos College of Physicians and Surgeons, Columbia University, New York, New York, ${ }^{2}$ Mortimer B. Zuckerman \\ Mind Brain Behavior Institute, Department of Neuroscience, Department of Biochemistry and Molecular Biophysics, Howard Hughes Medical \\ Institute, Vagelos College of Physicians \& Surgeons, Columbia University, New York, New York, and ${ }^{3}$ Department of Neuroscience, Kavli Institute \\ for Brain Science, Mortimer B. Zuckerman Mind Brain Behavior Institute, Department of Pharmacology, Vagelos College of Physicians and \\ Surgeons, Columbia University, New York, New York
}

Odors activate distributed ensembles of neurons within the piriform cortex, forming cortical representations of odor thought to be essential to olfactory learning and behaviors. This odor response is driven by direct input from the olfactory bulb, but is also shaped by a dense network of associative or intracortical inputs to piriform, which may enhance or constrain the cortical odor representation. With optogenetic techniques, it is possible to functionally isolate defined inputs to piriform cortex and assess their potential to activate or inhibit piriform pyramidal neurons. The anterior olfactory nucleus (AON) receives direct input from the olfactory bulb and sends an associative projection to piriform cortex that has potential roles in the state-dependent processing of olfactory behaviors. Here, we provide a detailed functional assessment of the AON afferents to piriform in male and female $\mathrm{C} 57 \mathrm{Bl} / 6 \mathrm{~J}$ mice. We confirm that the AON forms glutamatergic excitatory synapses onto piriform pyramidal neurons; and while these inputs are not as strong as piriform recurrent collaterals, they are less constrained by disynaptic inhibition. Moreover, AON-to-piriform synapses contain a substantial NMDAR-mediated current that prolongs the synaptic response at depolarized potentials. These properties of limited inhibition and slow NMDAR-mediated currents result in strong temporal summation of AON inputs within piriform pyramidal neurons, and suggest that the AON could powerfully enhance activation of piriform neurons in response to odor.

Key words: anterior olfactory cortex; anterior olfactory nucleus; associational synapses; intracortical synapses; olfactory cortex; piriform cortex

\section{Significance Statement}

Odor information is transmitted from olfactory receptors to olfactory bulb, and then to piriform cortex, where ensembles of activated neurons form neural representations of the odor. While these ensembles are driven by primary bulbar afferents, and shaped by intracortical recurrent connections, the potential for another early olfactory area, the anterior olfactory nucleus $(\mathrm{AON})$, to contribute to piriform activity is not known. Here, we use optogenetic circuit-mapping methods to demonstrate that AON inputs can significantly activate piriform neurons, as they are coupled to NMDAR currents and to relatively modest disynaptic inhibition. The AON may enhance the piriform odor response, encouraging further study to determine the states or behaviors through which AON potentiates the cortical response to odor.

Received Apr. 16, 2020; revised 0ct. 15, 2020; accepted 0ct. 20, 2020.

Author contributions: M.J.R., K.M.F., R.A., and S.A.S. designed research; M.J.R., K.M.F., and R.O. performed research; M.J.R. analyzed data; M.J.R. wrote the first draft of the paper; M.J.R. wrote the paper; K.M.F., R.A., and S.A.S. edited the paper.

This work was supported by the Howard Hughes Medical Institute to S.A.S. and R.A.

M. J. Russo's present address: Department of Neurology, New York University School of Medicine, New York, New York 10017.

K. M. Franks' present address: Department of Neurobiology, Duke University Medical School, Durham, North Carolina 27708.

R. Oghaz' present address: Department of Oral and Maxillofacial Surgery, Icahn School of Medicine at Mt. Sinai, New York, New York 10028

The authors declare no competing financial interests.

Correspondence should be addressed to Steven A. Siegelbaum at sas8@columbia.edu.

https://doi.org/10.1523/JNEUROSCI.0965-20.2020

Copyright $\odot 2020$ the authors

\section{Introduction}

The piriform cortex provides a simplified circuitry for studying cortical-sensory integration and the influences of intracortical information on cortical sensory representations. Within the piriform, odors activate unique and sparsely distributed ensembles of neurons, forming a cortical representation of odor (Poo and Isaacson, 2009; Stettler and Axel, 2009; Roland et al., 2017). These ensembles are formed in part by distributed inputs from the olfactory bulb, which drive powerful excitatory inputs onto piriform principal neurons (Franks and Isaacson, 2006; Suzuki and Bekkers, 2006, 2011; Davison and Ehlers, 2011). In addition, there is a significant contribution from intracortical or associative 
inputs to piriform (Poo and Isaacson, 2011). Piriform receives extensive associative inputs from diverse areas, which include other olfactory areas, such as the anterior olfactory nucleus (AON) or olfactory tubercle, higher-order areas, such as frontal or entorhinal cortex, or from the piriform itself in the form of recurrent collateral connections (Haberly and Price, 1978a; Datiche et al., 1996; Johnson et al., 2000; Franks et al., 2011). Optogenetic methods allow functional isolation of these various classes of inputs to piriform cortex and assessment of their roles in cortical odor processing.

The most comprehensively studied associative inputs to piriform cortex are the recurrent collateral synapses, which arise from other piriform pyramidal neurons and semilunar cells, and form a dense nexus of intracortical glutamatergic excitatory connectivity. Although these recurrent connections are spatially extensive, their excitation is constrained by strong, locally mediated feedback inhibition whose strength directly scales with excitation (Franks et al., 2011). This strong inhibition shapes the temporal integration of these associative inputs with the primary bulbar inputs. Moreover, the extensive recurrent network, when coupled with disynaptic inhibition, has been shown to constrain the spatiotemporal odor response to strong olfactory input, and thus provides a circuit-level mechanism for concentration-invariance of the odor representation (Bolding and Franks, 2018; Stern et al., 2018).

Here, we focus on another major class of associative inputs to piriform with potential to shape the cortical odor representations: the inputs from the AON. The AON is the earliest cortical olfactory area. It receives direct olfactory bulb input from mitral and tufted cells. AON neurons respond to multiple odors, and likely integrate convergent inputs from multiple olfactory bulb glomeruli (Lei et al., 2006). From prior anatomic studies (Haberly and Price, 1978b) and more recent optogenetic assays (Hagiwara et al., 2012), it is known that the AON provides significant excitatory input to the piriform cortex. Additionally, recent studies of the centrifugal projections from the AON to the olfactory bulb indicate that the AON preferentially synapses onto bulbar inhibitory neurons, and therefore drives significant inhibition onto mitral cells, which may dynamically filter signal-tonoise for odors according to cortical output (Boyd et al., 2012; Markopoulos et al., 2012). Moreover, the AON is potentially an important area for behaviorally relevant top-down control by neuromodulators or limbic circuits, and may be a critical mediator of state-dependent olfactory behaviors (Aqrabawi et al., 2016; Oettl et al., 2016).

We provide a functional isolation and quantitative characterization of AON projections to piriform, yielding insight into the potential influence of AON on piriform activity. Using viral vectors for focal and robust expression of channelrhodopsin-2 (ChR2) in the AON, we selectively activated AON afferents to piriform cortex (Boyden et al., 2005; Zhang et al., 2006; Yizhar et al., 2011). Through in vitro patch-clamp electrophysiology, we characterized the basic synaptic properties of AON synapses onto their piriform targets.

\section{Materials and Methods}

All experiments followed approved national and institutional guidelines of the Columbia University Medical Center. All animal experiments were conducted with male or female $\mathrm{C} 57 \mathrm{Bl} / 6 \mathrm{~J}$ mice from The Jackson Laboratory, and were reviewed and approved by the Institutional Animal Care and Use Committee.

Viral vector production and stereotaxic gene delivery. A Cre recombinase/GFP cassette (Le et al., 1999) was cloned into a lentivirus (LV) vector with expression driven by the humanized synapsin promoter
(hSyn1). High-titer LV was produced commercially (System Biosciences, $\sim 10^{9} \mathrm{IFU} / \mathrm{ml}$ ). Adeno-associated virus (AAV) for channelrhodopsin expression was generated from a pAAV-EF1 $\alpha$-DIO-hChR2(H134R)EYFP.WPRE.pA (gift from Karl Deisseroth) and produced commercially (UNC Vector; serotype 2/1). The recombinant AAV2/9 CAG-DIO-GFP and AAV2/1 CAG-DIO-TdTomato used for characterization of infection site and expression, and the AAV2/9 CamKIIa.hChR2(H134R)EYFP.WPRE.SV40 used for extensive channelrhodopsin expression in the AON were produced commercially and obtained from Penn Vector. All viruses were separated into $2.5-4 \mu \mathrm{l}$ aliquots and stored at $-80^{\circ} \mathrm{C}$ until just before injection.

Young adult male or female C57Bl/6J mice (4-8 weeks old) were anesthetized with ketamine/xylazine (100/10 mg/kg, respectively, i.p.) and head-fixed in a stereotaxic device. Virus was injected with a pulled glass pipette using standard procedures (Cetin et al., 2006). Briefly, an incision was made in the scalp and small craniotomy drilled above the AON using stereotaxic coordinates $2.8 \pm 0.17 \mathrm{~mm}$ anterior to bregma, $1.0 \pm 0.2 \mathrm{~mm}$ lateral, and depth $3.5 \pm 0.57 \mathrm{~mm}$, or above anterior piriform cortex with coordinates $0.5 \pm 0.6 \mathrm{~mm}$ anterior to bregma, $3.4 \pm 0.2$ $\mathrm{mm}$ lateral, and depth $5.3 \pm 0.1 \mathrm{~mm}$. Individual aliquots of $\mathrm{LV}$ and AAV were thawed, mixed (1:1 volume), and injected in $\sim 20 \mathrm{nl}$ increments using a Nanoject II (Drummond Scientific) through a glass pipette (Drummond Scientific, 3-000-203-G/X, tip diameter $\sim 20 \mu \mathrm{m}$ ). Volume injected was $612 \pm 246 \mathrm{nl}$ (range 200-760 nl, 19 animals) for the LVAAV combination, and $52.7 \pm 21.9 \mathrm{nl}$ (range $27-83 \mathrm{nl}, 12$ animals) for the nonconditional AAV2/9 CamKIIa.hChR2(H134R)-EYFP.WPRE. SV40. Only a single hemisphere (left or right, chosen randomly) was injected. The pipette was left in for $5 \mathrm{~min}$ before slow retraction. Off-target injections resulted in no expression or expression that was largely absent from the AON, and argue against spurious activation from other brain regions that were sometimes infected following virus injection. Animals with off-target infection were not included in these experiments.

Electrophysiology. After sufficient time for gene expression (typically 21-28 d after injection: $23 \pm 2.5 \mathrm{~d}$ for LV-AAV and $28 \pm 4 \mathrm{~d}$ for AAV9), mice were anesthetized with isoflurane and decapitated. The cortex was quickly removed in ice-cold aCSF. Horizontal brain slices $(350 \mu \mathrm{m})$ were cut using a vibrating microtome (Leica Microsystems) in a solution containing the following (in $\mathrm{mM}$ ): $10 \mathrm{NaCl}, 2.5 \mathrm{KCl}, 0.5 \mathrm{CaCl}_{2}, 7 \mathrm{MgSO}_{4}$, $1.25 \mathrm{NaH}_{2} \mathrm{PO}_{4}, 25 \mathrm{NaHCO}_{3}, 10$ glucose, and 195 sucrose, equilibrated with $95 \% \mathrm{O}_{2}$ and $5 \% \mathrm{CO}_{2}$. Slices were incubated at $34^{\circ} \mathrm{C}$ for $30 \mathrm{~min}$ in aCSF containing the following (in $\mathrm{mM}$ ): $125 \mathrm{NaCl}, 2.5 \mathrm{KCl}, 1.25$ $\mathrm{NaH}_{2} \mathrm{PO}_{4}, 25 \mathrm{NaHCO}_{3}, 25$ glucose, $2 \mathrm{CaCl}_{2}, 1 \mathrm{MgCl}_{2}$, and $2 \mathrm{Na}$ pyruvate. Slices were then maintained at room temperature until they were transferred to a recording chamber on an upright microscope (Olympus Optical) equipped with a $40 \times$ objective (LUMPLFLN $40 \mathrm{XW}, 0.8$ N.A.). For voltage-clamp recordings, patch electrodes $(3-6 \mathrm{M} \Omega)$ contained the following: 130 D-gluconic acid, $130 \mathrm{CsOH}, 5 \mathrm{~mm} \mathrm{NaCl}, 10$ HEPES, 12 phosphocreatine, 3 MgATP, 0.2 NaGTP, 10 EGTA, 0.05 AlexaFluor-594 cadaverine, $0.15 \%$ biocytin. For current-clamp recordings, electrodes contained the following: $130 \mathrm{~K}$-methylsulfonate, $5 \mathrm{~mm} \mathrm{NaCl}, 10$ HEPES, 12 phosphocreatine, 3 MgATP, $0.2 \mathrm{NaGTP}$, 0.1 EGTA, 0.05 AlexaFluor594 cadaverine, $0.15 \%$ biocytin. Voltage- and current-clamp responses were recorded with a Multiclamp $700 \mathrm{~A}$ amplifier, filtered at $2-4 \mathrm{kHz}$, and digitized at $10 \mathrm{kHz}$ (Instrutech). Series resistance was typically 8-12 $\mathrm{M} \Omega$, always $<20 \mathrm{M} \Omega$, and was compensated at $80 \%-95 \%$. The bridge was balanced using the automated Multiclamp function in currentclamp recordings. Short, collimated light pulses from a 470 nм LED (LEDC5, Thor Labs; $0-250 \mu \mathrm{W}$ measured at sample) were delivered to the tissue through the objective every 10-15 s. Most experiments used a $2 \mathrm{~ms}, 250 \mu \mathrm{W}$ pulse. We did not correct for liquid junction potentials. All experiments were performed at $34^{\circ} \mathrm{C}$.

Pharmacological agonists/antagonists CNQX (10 $\mu \mathrm{M})$, D-AP5 (50 $\mu \mathrm{M})$, SR95531 (10 $\mu \mathrm{M})$, CGP $55845(10 \mu \mathrm{M})$, TTX (1 $\mu \mathrm{M})$, and 4-AP $(100 \mu \mathrm{M})$ were all obtained from Tocris Bioscience.

Identification of layer 2 pyramidal neurons. Neurons were patched in deep layer 2 under differential interference contrast (DIC) optics and visualized at $590 \mathrm{~nm}$ with a monochrometer and cooled CCD camera (TILL Photonics). Pyramidal cells were identified based on morphologic 
and electrophysiological indicators (Suzuki and Bekkers, 2006); that is, one or two spiny apical dendrites that branched extensively and extended to layer 1a, basal dendrites that extended into layer 3 , and input resistances of 100-200 $\mathrm{M} \Omega$. Recordings from semilunar cells or high input resistance layer 2 interneurons could readily be distinguished from pyramidal cells and were not included in this analysis. After recording, brain slices were fixed and viewed by fluorescence confocal microscopy to further confirm that patched cells were layer 2 pyramidal neurons.

Laser-scanning photostimulation and synaptic input mapping. Laserscanning photostimulation was performed by scanning the slice surface with a pair of galvanometer-controlled scan mirrors (TILL Photonics), controlled by custom software written in C and LabView (National Instruments). The $470 \mathrm{~nm}$ laser (100 mW) was power modulated with an acoustic-optical tuning filter (AA Optoelectronic) and fiber-coupled to the scan mirrors. Planar deflection was achieved with a scan lens (TILL Photonics) behind the objective. The beam diameter $\left(1 / \mathrm{e}^{2}\right)$ of the illumination spot was either $\sim 30 \mu \mathrm{m}(20 \times$ objective $)$ or $\sim 15 \mu \mathrm{m}(40 \times$ objective). The order of site illumination was initially designed so as to maximize the time between illumination of neighboring sites, thus minimizing artifact from inactivation by neighboring illumination. However, serial trials with conventional illumination pattern (left-to-right, top-tobottom) showed no such neighborhood effects, and these data were pooled.

Comparisons with piriform recurrent inputs. Synaptic response data from our prior study of piriform recurrent collaterals were included only for comparison of AON responses within piriform (see Figs. 2, 4). These data were obtained identically to the methods outlined above, with the exception that dual viral vectors were injected into piriform cortex for expression in anterior piriform cortical neurons (Franks et al., 2011).

Data analysis. Data were collected and analyzed offline using a combination of Axograph X (Axograph), IGOR Pro (Wavemetrics), MATLAB (The MathWorks), and Prism (GraphPad). Average responses were calculated from 12-20 consecutive episodes. Synaptic time constants were determined from a monoexponential best fit when appropriate. Half-width duration was used as a metric of synaptic time course when the response was only well fit by a multiexponential process, as in the case of rapid attenuation of EPSPs by inhibition. Synaptic conductance $\left(G_{\text {excitation }}\right.$ or $\left.G_{\text {inhibition }}\right)$ was calculated from $G=P S C /\left(V_{\text {hold }}-\right.$ $\left.E_{\text {rev }}\right)$ where reversal potentials $E_{\text {rev }}$ were theoretical and calculated from the ionic compositions of the intracellular and extracellular solutions $\left(E_{\text {rev, excitation }}=E_{\text {rev, }}, N_{M D A}=5 \mathrm{mV}\right.$ and $\left.E_{\text {rev, inhibition }}=-92 \mathrm{mV}\right)$. Statistical tests were unpaired or paired two-sided $t$ tests, as appropriate, except where data were clearly not normally distributed, as in EPSC amplitudes in across neurons, in which case Mann-Whitney $U$ test (i.e., Wilcoxon rank sum test) was applied. Each applied statistical test is indicated in the text and/or figure legends.

\section{Results}

\section{Robust, focal expression of ChR2 within the AON}

The AON is within the olfactory peduncle, the thin stalk of tissue that joins the olfactory bulb to the forebrain. It is nearly continuous with the olfactory bulb, the piriform cortex, and the orbitofrontal cortical areas, and abuts the dense fasciculation of axons of the lateral olfactory tract (LOT) that carries information from bulb to other forebrain areas (Brunjes et al., 2011). We used a previously established (Choi et al., 2011; Franks et al., 2011) dual viral vector approach to achieve strong expression of ChR2 in a spatially restricted subpopulation of AON neurons (Fig. 1A). Simultaneous injection of an LV vector expressing Cre recombinase (LV-Cre) and an AAV vector containing a Cre-dependent $\mathrm{ChR} 2$ construct into the AON resulted in focal, sparse and strong expression of ChR2 in the $\mathrm{AON}$, as observed with previous experiments in piriform cortex (Fig. 1B) (Franks et al., 2011).

Focal expression of ChR2-YFP in the AON revealed YFPpositive $\left(\mathrm{YFP}^{+}\right)$axons that coursed along the rostrocaudal extent of anterior piriform cortex (the portion of piriform directly bordered by the LOT), but their density tapered as they entered the posterior piriform (Fig. 1C). This is consistent with prior tract-tracing studies that showed a similar sharp attenuation of labeled AON axons at the border of posterior piriform (Haberly and Price, 1978b). Additionally, $\mathrm{YFP}^{+}$AON axons were consistently seen in the olfactory bulb, as well as the contralateral olfactory bulb, contralateral $\mathrm{AON}$, and contralateral anterior piriform (not shown), also consistent with known projections (Illig and Eudy, 2009; Markopoulos et al., 2012). Within piriform, AON axons fasciculated within layer $1 \mathrm{~b}$, similarly to recurrent collateral axons from piriform pyramidal neurons (Fig. 1D), and ramified throughout layer 3 . There were also a few $\mathrm{YFP}^{+}$axons within layer 1a, where bulbar axons synapse onto distal dendrites of piriform pyramidal neurons and where interneurons are located that receive bulbar input and mediate feedforward inhibition onto piriform neurons (Luna and Schoppa, 2008; Stokes and Isaacson, 2010; Suzuki and Bekkers, 2012). Although sparse, fibers within layer 1a were consistently seen when ChR2-YFP was expressed in the AON, and have also been observed using traditional tract-tracing techniques (Haberly and Price, 1978b). Fluorescent fibers were not observed in the LOT.

We recorded from $\mathrm{YFP}^{+}$neurons in the AON to verify strong $\mathrm{ChR} 2$ expression. In a cell-attached configuration, trains of blue light pulses reliably evoked action potential firing in $\mathrm{YFP}^{+}$neurons, while whole-cell recordings revealed large, excitatory photocurrents $(-1.81 \pm 0.86 \mathrm{nA}$, mean $\pm \mathrm{SD}, n=7$, not shown), with time course characteristic of ChR2-mediated inward currents (Hegemann et al., 2005; Zhang et al., 2006).

We sought additional confirmation that we reliably and exclusively targeted the AON for exogenous protein expression with our methods by coinjecting LV-Cre- and Cre-dependent $\mathrm{AAVs}$ driving fluorescent markers into the $\mathrm{AON}$ and piriform cortex in the same animal (Fig. 1E). We used expression of GFP or TdTomato to provide a strong cytosolic signal that more clearly indicates cell bodies and dendrites than was seen with ChR2-YFP expression because of its membrane-targeting. Injection of LV-Cre and AAV2/9-CAG-DIO-GFP into the AON and LV-Cre and AAV2/1-CAG-DIO-TdTomato into the piriform produced spatially restricted expression of the appropriate fluorophores (Fig. $1 F, G$ ). While $\mathrm{GFP}^{+}$and TdTomato ${ }^{+}$axons were faintly visible in both areas, there was no overlap in the populations of $\mathrm{GFP}^{+}$or TdTomato ${ }^{+}$neurons (Fig. $1 H, I$ ). $\mathrm{GFP}^{+}$ axons had similar distribution to ChR2-YFP-expressing axons across piriform layers (Fig. 1J). We observed a similarly restricted expression pattern when the location of the fluorescent vector injection was reversed, with AAV2/1-CAG-DIO-TdTomato injected into AON and AAV2/9-CAG-DIO-GFP injected into piriform (not shown).

\section{Light-evoked synaptic responses from AON inputs to piriform}

With robust ChR2 expression restricted to the $\mathrm{AON}$, we assessed the properties of AON afferents to piriform cortex. We stimulated with wide-field illumination, with the objective positioned over the soma of the patch-clamped neuron, to evoke maximal EPSC responses at a membrane voltage of $-70 \mathrm{mV}$. Morphology and identity of recorded neurons were verified by direct visualization during recording and in post hoc confocal microscopy of fixed brain slices. Brief ( $2 \mathrm{~ms}$ ) light pulses evoked rapid inward currents in whole-cell recordings from piriform layer 2 pyramidal neurons with the membrane held at $-70 \mathrm{mV}$ (Fig. $2 A, B$ ), which were highly consistent from trial to trial. Median latency and jitter for these responses were 3.0 and $0.16 \mathrm{~ms}$, respectively, 
A



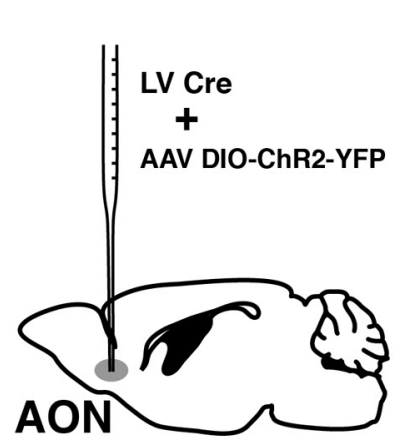

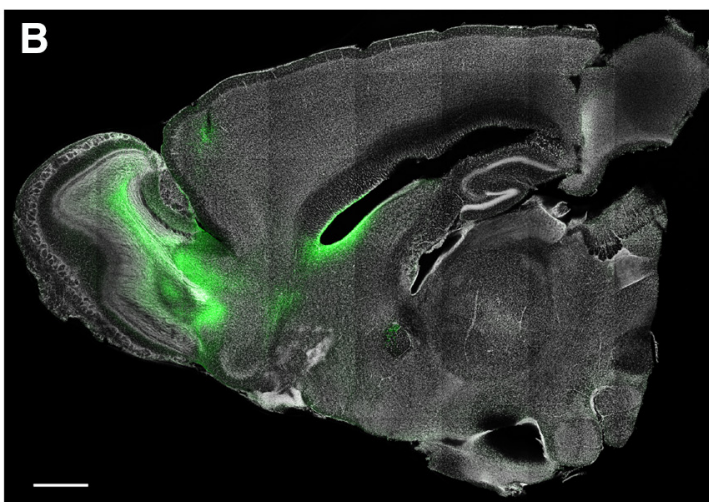

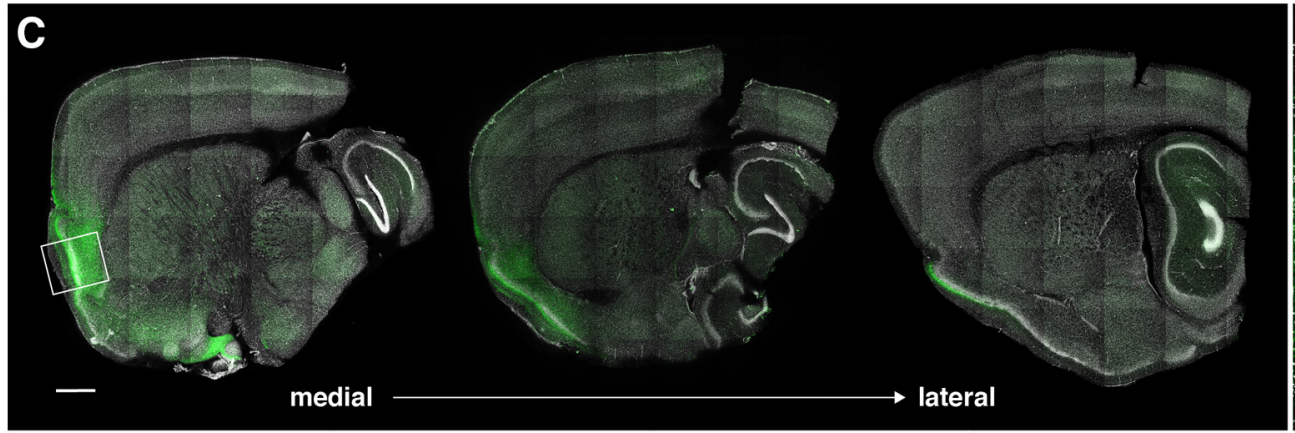
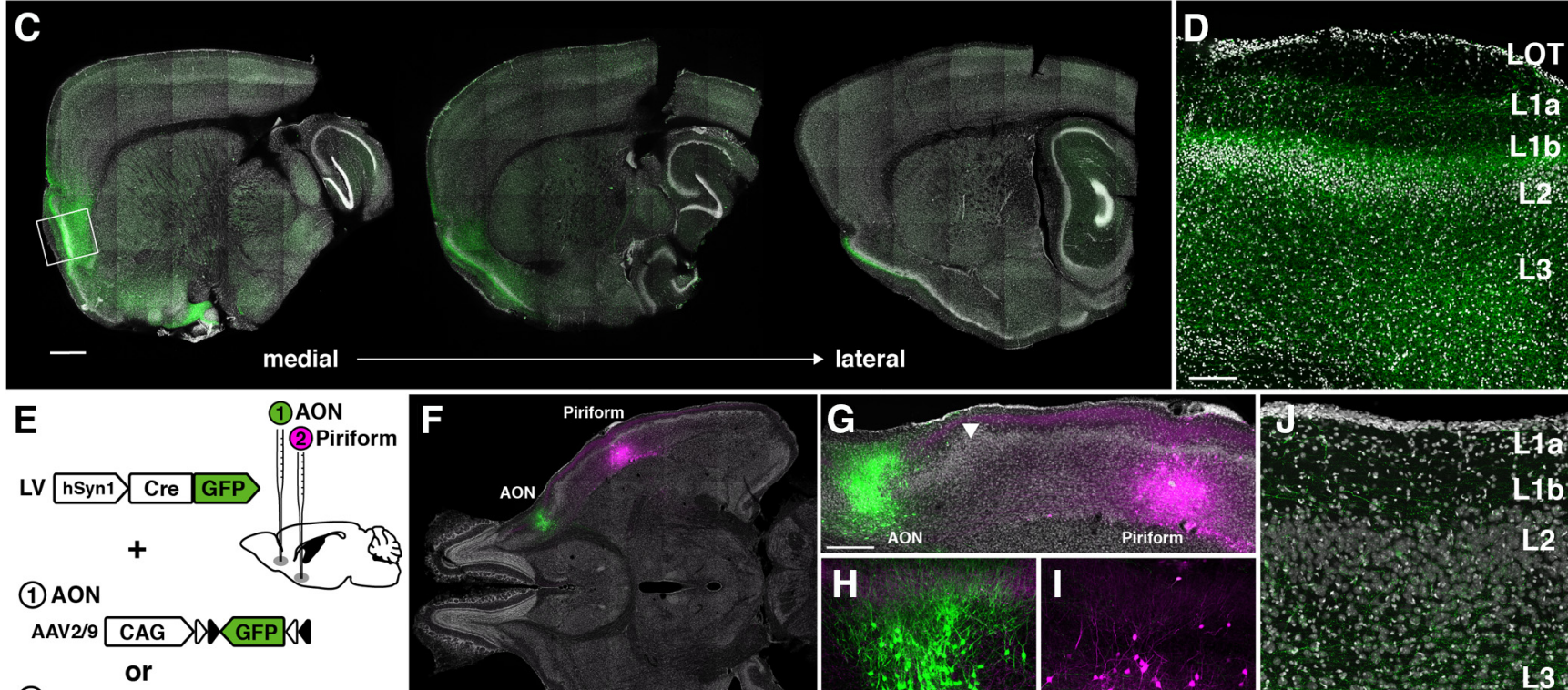

(2) Piriform
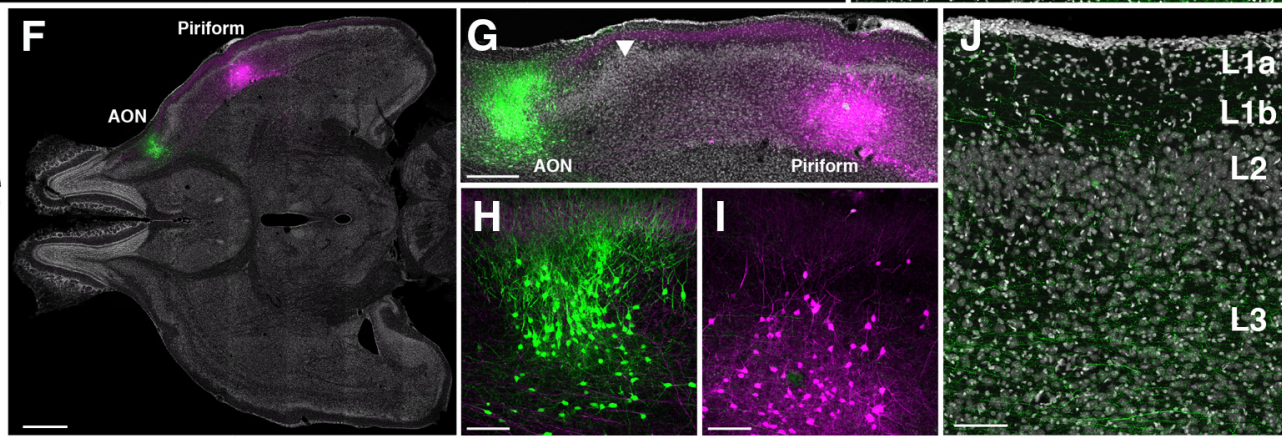

Figure 1. Focal expression of ChR2 within the AON. A, Viral vectors and gene constructs used for strong, localized expression of ChR2 in the AON. LV for Cre recombinase expression in a sparse, focal set of neurons, and AAV for expression of Cre-dependent ChR2-YFP. Right, These viral vectors were simultaneously injected into the central AON within the olfactory peduncle. The LV construct contains the humanized synapsin promoter (hSyn1) for pan-neuronal expression, and the Cre-dependent AAV construct includes humanized elongation factor $1 \alpha$ (EF1 $\alpha$ ) for constitutive ChR2 expression in all neurons expressing Cre recombinase. LoxP and Lox2272 refer to independent pairs of site-specific Cre recombination sequences. $\boldsymbol{B}$, Parasagittal section of an injected mouse forebrain showing ChR2-YFP expression well localized to the AON. Scale bar, $1 \mathrm{~mm}$. C, Visualization of AON projections to piriform cortex. Medial-lateral series of parasagittal sections from an injected brain. Y Y ${ }^{+}$axons course ventrolaterally from the AON to the piriform. Axon density tapers sharply after anterior piriform, and axons do not densely innervate the posterior piriform. Scale bar, $1 \mathrm{~mm}$. D, Laminar distribution of AON afferents to piriform. Magnified view (rotated) of the boxed area in C, showing YFP ${ }^{+}$axons in the anterior piriform cortex. Scale bar, $100 \mu \mathrm{m}$. Fibers are densest in layer $1 \mathrm{~b}$, less dense in in layer 3, and occasionally seen in layer 1a (but no fibers are seen within the LOT). $\boldsymbol{E}$, Verification that vector-mediated expression was restricted to a subset of AON neurons. Using a similar dual LV-AAV vector strategy, Cre-dependent GFP was expressed in the AON, while Cre-dependent TdTomato was expressed in piriform in the same animal via two separate injections. Identical volumes and titers of LV-Cre vector were used as for channelrhodopsin experiments. $\boldsymbol{F}$, Horizontal section of forebrain showing ipsilateral injection sites with expression of GFP in a restricted area of AON, and similarly restricted expression of TdTomato in the piriform cortex. Scale bar, $1 \mathrm{~mm}$. G, Enlarged view of lateral forebrain, including both injection sites, showing GFP ${ }^{+}$(green) and TdTomato ${ }^{+}$(magenta) axons in both areas, but no overlap in green or red fluorescent somata. Scale bar, $200 \mu \mathrm{m}$. Arrowhead roughly indicates AON-piriform transition area. $\boldsymbol{H}, \boldsymbol{I}$, Enlarged views of the respective injection sites above, demonstrating sparse and focal expression of Cre-dependent fluorescent proteins in both the AON and piriform. Scale bars, $50 \mu \mathrm{m}$. J, Area of piriform cortex between injection sites. GFP ${ }^{+}$axons from AON neurons projecting through piriform layers with similar distribution to $\mathrm{YFP}^{+}$axons of ChR2-expressing AON neurons in $\boldsymbol{D}$. TdTomato fluorescence not shown. LOT not visible in this section. Scale bar, $100 \mu \mathrm{m}$.

consistent with single-synapse transmission (Fig. 2B, right). Light-evoked responses were also consistently and reversibly abolished by blockade of fast glutamatergic transmission (Fig. $2 C)$. NMDAR-mediated synaptic currents were isolated and measured by blocking both inhibition and fast, AMPAR-mediated currents, and by voltage-clamping neurons at highly positive potentials $\left(40-50 \mathrm{mV}\right.$ ) to relieve $\mathrm{Mg}^{2+}$ block. We found that AON synapses onto piriform pyramidal neurons evoked a large NMDAR-mediated synaptic current (Fig. 2D, mean NMDA: AMPA conductance ratio was $7.5 \pm 2.7$, mean \pm SEM, $n=16$ ). These data indicate that the AON makes monosynaptic, glutamatergic synapses onto piriform pyramidal neurons, and that these synapses have significant NMDAR-mediated currents, consistent with prior physiological studies of AON-to-piriform afferents (Kanter and Haberly, 1990; Hagiwara et al., 2012). Moreover, the large NMDAR component of the EPSC is similar to that observed at piriform recurrent collateral synapses (Fig. 2D) (Franks and Isaacson, 2005).

We quantified the range of AON synaptic responses by recording from 121 visually identified layer 2 piriform pyramidal neurons from 16 animals in which ChR2 was expressed in the AON. Although large EPSCs were observed on occasion 
(-786 pA in the example of Fig. 2E, top), the responses were typically much smaller (-65.9 pA, Fig. 2E, middle), and were often on the order of the unitary response to activation of a single AON fiber $(-11.2 \mathrm{pA}$, Fig. 2E, bottom; see Fig. $2 F$ ). The median response was $-47.1 \mathrm{pA}$ (range $-4.5 \mathrm{pA}$ to $-786 \mathrm{pA}$ ), and the mean response was $-96.2 \pm 146 \mathrm{pA}$ (mean $\pm \mathrm{SD}, n=66$ ). Of the 121 piriform neurons assessed, only 66 (55\%) demonstrated a detectable response to light, in contrast to the $99 \%$ probability of observing a response following excitation of the recurrent network under similar experimental conditions (Franks et al., 2011).

In a limited number of recordings, we found that four of four semilunar neurons and four of six layer 3 pyramidal neurons received excitatory input from the AON. However, we did not record from sufficient numbers to meaningfully assess the relative strength or frequency of $\mathrm{AON}$ inputs to these neuron types, and have focused our analysis on AON connections to piriform layer 2 pyramidal neurons. A prior study found that the AON makes excitatory connections onto both semilunar and layer 2 and 3 pyramidal neurons, but connections to semilunar neurons were relatively weak compared with those onto pyramidal neurons (Hagiwara et al., 2012).

We next measured the unitary excitatory response amplitude (unitary EPSC) for AON-to-piriform afferent synapses to determine whether any clustering of synapses occurs and whether the AON is capable of producing strong, single-fiber responses onto piriform neurons, in a manner similar to primary bulbar afferents from the LOT (Franks and Isaacson, 2006). Stimulation with minimal light power and spatially restricted illumination elicited discrete responses with alternating successes and failures (perithreshold stimulation). The unitary ESPCs recorded with this method were $-14.9 \pm 1.7 \mathrm{pA}$ (mean \pm SEM, $n=9$; Fig. $2 F$ ). This is on the order of the spontaneous miniature EPSC responses recorded in these cells, suggesting that a single AON afferent makes only a single terminal or en passant synapse onto a piriform pyramidal neuron, similar to findings for piriform recurrent collaterals (Franks et al., 2011), although the response to a unitary $\mathrm{AON}$ input is somewhat weaker, equal to $\sim 40 \%$ that of piriform recurrent synapses (Fig. $2 F$, right; $-36.2 \pm 5.9 \mathrm{pA}$ ) (Franks et al., 2011).

The short-term dynamics of AON-topiriform synapses were assessed by eliciting pairs of EPSCs at various time intervals and measuring the facilitation or depression of the second EPSC in the pair (Fig. 2G). We
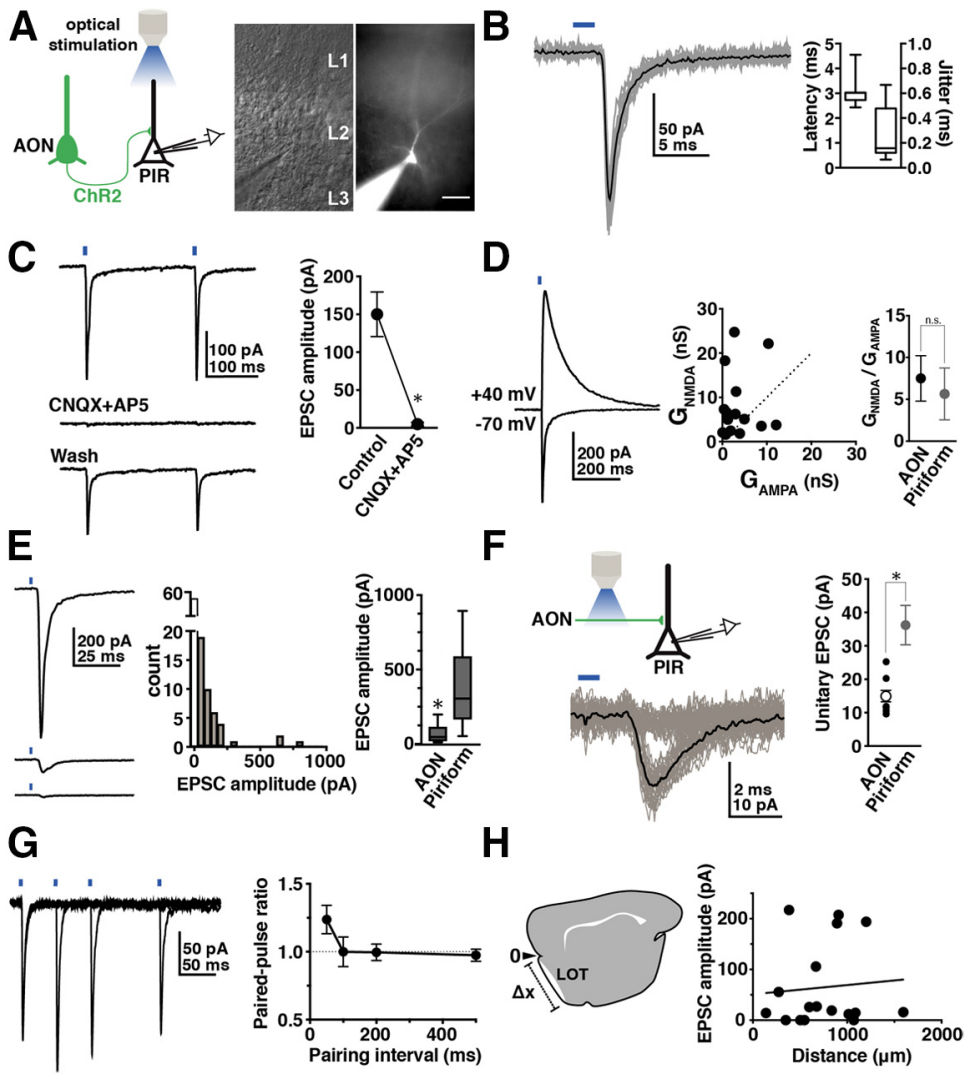

H

$\mathbf{F}$
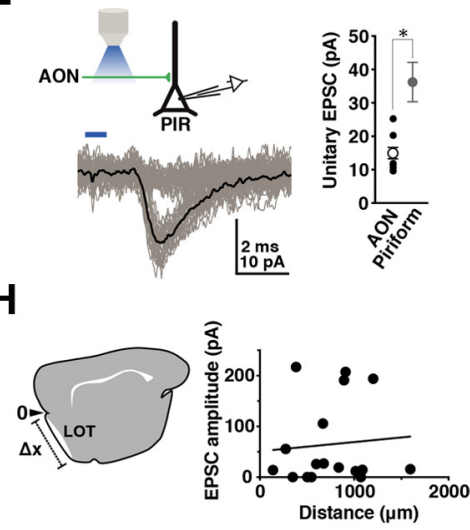

Figure 2. Photoactivation of AON afferents to piriform reliably evokes excitatory responses in piriform pyramidal neurons. A, Recording from layer 2 pyramidal neurons in piriform, while photoactivating afferents originating in AON. Cells were targeted under DIC (middle), and morphology was visualized after filling with intracellular Alexa-594 fluorescent dye (right). Scale bar, $50 \mu \mathrm{m}$. L1-L3 indicate piriform layers 1-3, respectively. B, Voltage-clamp recordings (holding at $-70 \mathrm{mV}$ ) from a piriform pyramidal neuron and responses to brief ( $2 \mathrm{~ms}$ ) light pulses, which are represented by the blue bar. Twelve consecutive stimulation trials are shown (gray traces) with the average response (black trace). Right, Plot of latency (left bar) and jitter (right bar, SD of latency) for 7 responses. For each, median (middle line), 25-75 percentiles (box edges), and 10-90 percentiles are shown (whiskers). Median latency: $3.0 \mathrm{~ms}$ (range 2.4-4.6 ms); median jitter: 0.16 ms (range $0.07-0.67 \mathrm{~ms}$ ). C, Responses to pairs of light pulses at $-70 \mathrm{mV}$ under control conditions and in the presence of blockers of glutamatergic synaptic transmission, CNQX and AP5. Responses return after washout of drugs (bottom). Right, EPSC amplitude of excitatory responses in absence (control) and presence of CNQX and AP5 (control: $150.3 \pm 12.2 \mathrm{pA}$; CNQX + AP5: $5.2 \pm 1.0 \mathrm{pA}$; mean \pm SEM, $n=5$ ). $* p<0.001$ (paired $t$ test). $\boldsymbol{D}$, NMDAR current associated with AON afferents to piriform. Left, EPSCs recorded at $-70 \mathrm{mV}$, and at $40 \mathrm{mV}$ to maximize NMDA-mediated currents (in the presence of blockers of fast and slow GABAergic transmission, SR79331 and CGP55845, respectively, and the AMPA-receptor blocker, $C N Q X)$. Middle, Plot of NMDA conductance $\left(G_{\text {NMDA }}\right)$ versus AMPA conductance $\left(G_{\text {AMPA }}\right)$ in each of 14 neurons. Right, NMDA:AMPA conductance ratios $\left(G_{N M D A} / G_{A M P A}\right)$ for AON-to-piriform afferents $(7.5 \pm 2.7$, mean $\pm S E M, n=14)$, with the NMDA:AMPA conductance ratio for piriform-to-piriform afferents (gray) for comparison (5.6 $\pm 3.1, n=10$, $p=0.66$, n.S., not significant, unpaired $t$ test). $\boldsymbol{E}$, Distribution of maximal AON inputs to piriform neurons. Left, Synaptic responses within 3 different neurons to show range of total EPSC size in these neurons. Peak (negative) amplitudes: bottom, $11.2 \mathrm{pA}$; middle, $65.9 \mathrm{pA}$; top, $788 \mathrm{pA}$. Middle, Histogram of maximum EPSC responses recorded in 66 piriform neurons. Right, Bar plot representing median response amplitude (middle), 25-75 percentiles (box edges), and 10-90 percentiles (whiskers). Median A0N-to-piriform response: $47.1 \mathrm{pA}$ (range 4.51-786pA); mean response $96.2 \pm 146 \mathrm{pA}$ (mean $\pm \mathrm{SD}$ ). Similar statistics are provided for piriform-to-piriform responses in 93 neurons: median piriform-to-piriform response was $305.0 \mathrm{pA}$ (range 18.0-1688 pA); mean response $414 \pm 335 \mathrm{pA}$ (mean $\pm \mathrm{SD}$ ). Median AON-to-piriform versus piriform-to-piriform -47.1 versus -305.0 pA. $* p<0.0001$ (Mann-Whitney $U$ test). $\boldsymbol{F}$, Unitary responses to A0N inputs onto piriform cells. Axons were stimulated with illumination at a site distant $(\sim 400 \mu \mathrm{m})$ from recorded cells. Left, Representative overlay of 20 trials, in which response failures occurred on $\sim 50 \%$ of the trials (perithreshold). Right, Plot of unitary response amplitudes in 9 cells $(-14.9 \pm 1.7 \mathrm{pA}$, mean $\pm \mathrm{SEM}, n=6)$. The unitary response determined similarly for piriform recurrent collaterals is provided for comparison (36.2 $\pm 5.9 \mathrm{pA}$, mean $\pm \mathrm{SEM}, n=12)$. $* p=0.0003$ (Mann-Whitney $U$ test). G, Pairing of light stimuli at varying time intervals to assess short-term synaptic plasticity. Left, Overlay of pairs of EPSCs evoked by light stimuli separated by $\Delta t=50,100$, and $200 \mathrm{~ms}$, and plot of paired-pulse ratio (second response/first response) for various pairing intervals (nonsignificant trend toward facilitation at $50 \mathrm{~ms}$ pairing, ratio $1.24 \pm 0.10$, mean \pm SEM $n=6, p=0.07$, paired $t$ test). $\boldsymbol{H}$, EPSC amplitude plotted as function of distance within anterior piriform cortex, indicating no apparent distance dependence of responses (pooled data from 3 brain slices from 3 different animals). Zero indicates AON-piriform transition area. 
found that AON afferent synapses were weakly facilitating at short intervals, with $\sim 25 \%$ facilitation at $50 \mathrm{~ms}$ pairing (pairedpulse ratio, second EPSC/first EPSC: $1.24 \pm 0.10$, mean \pm SEM, $n=6, p=0.07$, not significant), indicating a minor increase of release probability during the second pulse.

We observed responses of varying amplitudes throughout the extent of anterior piriform cortex. There was no increase in likelihood of observing larger responses within the rostral-most portions of anterior piriform. In pooled data from three slices from 3 separate animals, large EPSCs were observed 1-2 mm into anterior piriform cortex (Fig. $2 \mathrm{H}$, zero corresponds to AON-piriform transition area). We did not observe responses in any of 18 pyramidal neurons recorded in the posterior piriform (area of piriform caudal to the LOT termination).

Functional topography of AON afferents to piriform cortex The layer-specific topography of AON afferents to the piriform cortex was previously studied with classic tract-tracing methods (Haberly and Price, 1978b), with results consistent with the pattern of $\mathrm{YFP}^{+}$fluorescent axons that we observed (see Fig. 1D). We sought to determine whether this corresponds to a functional bias of AON synapses for the proximal apical dendritic domain of piriform pyramidal neurons, or whether significant activation may arise from AON fibers in layer 3, or even the sparse fibers observed in layer 1a (i.e., the distal dendritic domain). We used an established method to precisely map single AON synaptic inputs onto piriform pyramidal neurons (Petreanu et al., 2009). With a $470 \mathrm{~nm}$ laser directed by scanning mirrors, we illuminated highly focused areas within the brain slice along the somatodendritic axis of piriform pyramidal neurons (Fig. $3 A$ ). We included TTX in the bath to block action potentials to restrict photoactivation to synaptic terminals and included 4-AP to block potassium channels and enhance the local ChR2-mediated depolarization. A grid pattern of illumination was chosen to completely sample the apical and basal fields, from the LOT to deep layer 3, of the recorded neuron at center (Fig. $3 A$, right). Stimulation points were horizontally and vertically separated by $80 \mu \mathrm{m}$. An exemplary set of responses is shown in Figure $3 B$. Here, synaptic responses (or absence of response) are plotted at the corresponding point in the stimulus grid that evoked the response. A spatial convolution of the response amplitudes is generated for visualization (Fig. 3B, right).

We scanned the dendritic fields of 12 neurons, with measurements of EPSC amplitude at each site, and aligned these maps so that data from 12 neurons could be combined into an average intensity profile (actual scan area was larger than that shown here to allow alignment without artifact, and negative responses at the periphery were omitted). Piriform neurons were most strongly activated by stimulation of $\mathrm{AON}$ inputs over the proximal apical dendritic field in layer $1 \mathrm{~b}$ (Fig. 3C). The maximal response for each of the 12 neurons tested is indicated at its corresponding location in a single grid, which shows that most maximal responses occurred in the apical field of the neuron, and that these also tended to be much larger in EPSC amplitude than responses in the basal field (Fig. 3D). There are several neurons with responses in the basal field or layer 3, which suggest some degree of input from the layer 3 fibers seen in Figure $1 D$. There were no responses observed with focal illumination over layer 1a, the bulbar input layer.

\section{Weak disynaptic inhibition driven by AON afferents}

The AON provides excitatory input to piriform that is relatively weak compared with piriform recurrent collateral input. However, although the piriform recurrent collaterals evoke a large EPSP, the excitation is normally constrained by an even larger disynaptic IPSP (Franks et al., 2011). To determine whether the AON also drives strong disynaptic feedforward inhibition onto piriform neurons, we performed voltage-clamp recordings of piriform neurons while holding the membrane potential at $-70 \mathrm{mV}$ and at +5 to $+10 \mathrm{mV}$ to assess the relative strengths of excitatory and inhibitory responses to light activation, respectively (Fig. $4 A$ ). Brief light pulses evoked negative EPSCs at $-70 \mathrm{mV}$, as expected. While holding at $+5 \mathrm{mV}$ in the same neuron, we consistently observed a positive current in response to light, with delayed onset relative to the EPSC. The median latency and jitter for these positive current responses were 4 and $0.25 \mathrm{~ms}$, respectively, and suggest a polysynaptic process (Fig. 4A, right). Responses recorded at $+5 \mathrm{mV}$ were abolished by SR95331, an antagonist of $\mathrm{GABA}_{\mathrm{A}}$ receptors (reduced to $3.3 \pm 8 \%$ of the control response, $n=5$; data not shown). Moreover, addition of blockers of fast excitatory synaptic transmission (CNQX and AP5) completely and reversibly abolished these positive responses (Fig. 4B), indicating that they correspond to disynaptic inhibition. In contrast to results with stimulation of piriform collaterals, optogenetic activation of the AON inputs evoked an outward current at $5 \mathrm{mV}$ that was consistently smaller than the inward EPSC response at $-70 \mathrm{mV}$. A plot of the inhibitory conductance $\left(G_{\text {inhibitory }}\right)$ versus excitatory conductance $\left(G_{\text {excitatory }}\right)$ recorded from each cell in response to photoactivation of $\mathrm{AON}$ inputs revealed an inhibition-to-excitation conductance ratio of $0.71 \pm 0.24$ (mean \pm SEM, $n=29$; Fig. $4 C$ ), indicating that excitation is larger than inhibition. This differs markedly from synaptic responses to piriform collateral stimulation, where inhibition dominates.

It is possible that the relatively small excitatory responses to AON photostimulation underestimates the true extent of disynaptic inhibition relative to monosynaptic excitation. Because our experimental protocol involved expressing $\mathrm{ChR} 2$ in a relatively small subset of the AON population, this may have led to the inefficient excitation of local inhibitory neurons. We therefore asked whether a more extensive activation of the AON afferents could produce a larger inhibitory-to-excitatory response ratio. We expressed $\mathrm{ChR} 2$ in a larger fraction of $\mathrm{AON}$ neurons using a viral vector with a nonconditional AAV, with serotype 2/ 9 that has wider tissue spread (Fig. $4 D, E$ ). This method also produced larger photocurrents in AON neurons than with the focal method $(3.04 \pm 1.86 \mathrm{nA}$ vs $1.81 \pm 0.86 \mathrm{nA}$, mean $\pm \mathrm{SD}, n=6$, not shown), indicating higher efficiency of expression within a given cell. As this serotype has limited or no tropism for olfactory bulb mitral cells (Hagiwara et al., 2012), contamination by any bulbar projections is unlikely (Fig. $4 F$ ). Importantly, the nonconditional vector injection into AON produced a similar pattern of fluorescent axons within piriform to that seen with the focal, dual vector method, suggesting that the two methods recruit qualitatively similar populations of AON neurons (Fig. 4G; see Fig. 1D).

More extensive ChR2 expression in the AON led to larger light-evoked EPSCs in piriform pyramidal neurons voltageclamped at $-70 \mathrm{mV}$, as anticipated (Fig. $4 \mathrm{H}$ ). The median EPSC amplitude increased to $-139.9 \mathrm{pA}$ from $-47.1 \mathrm{pA}$ measured with more focal expression (mean value increased to $-260.8 \pm$ $307 \mathrm{pA}$ from $96.2 \pm 146 \mathrm{pA}$, mean $\pm \mathrm{SD}$ ), with a similar distribution, indicating a uniform increase in excitatory inputs (Fig. 4I). 
A
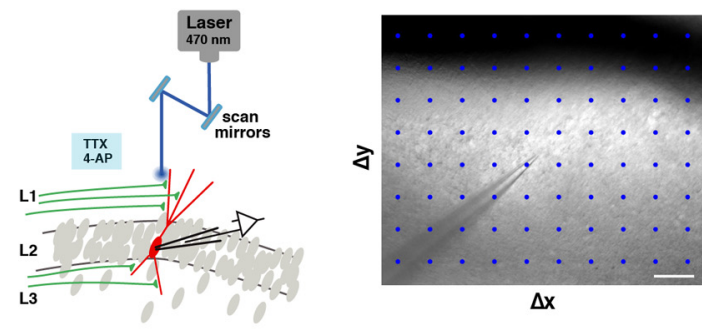

$\Delta \mathbf{x}$
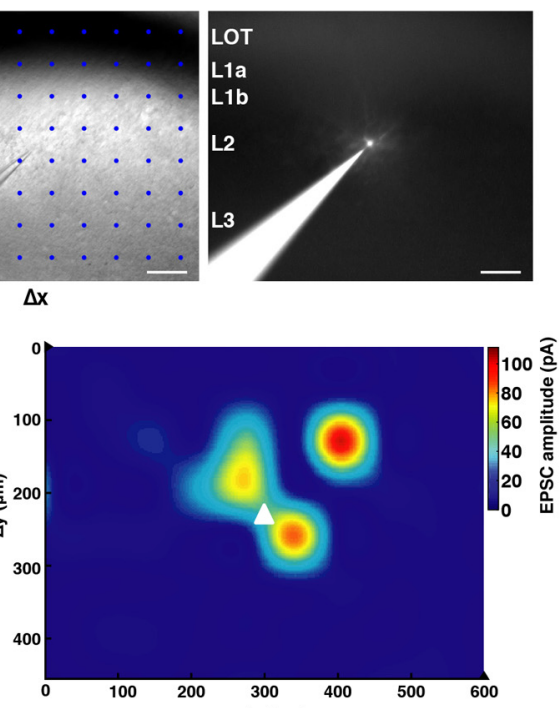

D



Figure 3. Laminar organization of AON inputs to piriform pyramidal neurons. $\boldsymbol{A}$, Left, Schematic of laser scanning activation of individual synaptic boutons. Using a scanning mirror and lens system, focal areas of the brain slice were activated in a grid pattern. Blockers of action potential propagation (TTX and 4-AP) were included to limit activation to areas on or near synaptic boutons. Middle, DIC image $(10 \times)$ showing a typical laser-scanning activation preparation. Stage position adjusted so that recorded cell is at center of the scan field. The center of scan field (blue spots, overlay) is chosen so as to cover the major input layers to the piriform cell ( $8 \times 10$ positions, $\Delta x=\Delta y=80 \mu \mathrm{m})$. Scale bar, $100 \mu \mathrm{m}$. Right, Fluorescent image of dye-filled cell to confirm identity and soma position. $\boldsymbol{B}$, Representative spatial response profile for a piriform pyramidal neuron. Left, Single scan of the synaptic inputs to the cell in $\boldsymbol{A}$. Response locations are indicated by their position in the grid. Color represents response amplitude (top right, color bar). Relative position of cell body is indicated by schematic pyramidal neuron in gray. Right, Interpolated intensity profile of the synaptic inputs for visualization. C, Average of intensity profile for all neurons tested. Each map was normalized to the maximal response for each neuron, aligned to somatic position, and these were averaged to produce the map shown ( $n=12$ neurons). $\boldsymbol{D}$, Locations and amplitudes of maximal responses during activation scans. Each intersection in the grid corresponds to a point of activation as in $\boldsymbol{B}$. Each colored marker represents the point of maximal activation for each of 12 neurons (i.e., each marker is separate scan experiment), with colors representing relative EPSC amplitude. Clustered markers represent points that produced maximal activation in multiple experiments. Black triangle represents location of somata of recorded neurons in all experiments.

The probability of recording an excitatory response of any amplitude also increased to $82 \%$ (58 total attempts, Fig. $4 J$ ). Thus, the more extensive ChR2 expression in the $\mathrm{AON}$ resulted in stronger AON-driven excitation in piriform.

We then asked whether this increased excitation led to an even larger increase in disynaptic inhibition, to levels similar to that seen with excitation of the piriform collaterals. Again, inhibitory conductance was determined as a function of excitatory conductance for each voltage-clamped neuron from which we recorded (Fig. $4 K$ ). In this set of experiments, there were several neurons with substantial excitation $(\sim 10 \mathrm{nS}$ excitatory conductance) and one neuron with $\sim 20 \mathrm{nS}$ excitation. However, the inhibition-to-excitation ratio remained low $(1.17 \pm 0.33$, mean \pm SEM, $n=19$ ), similar to that noted above with more focal ChR2 expression (Fig. $4 L ; p=0.27$ for comparison with focal I:E ratio; unpaired $t$ test). As further confirmation that we were maximally activating inhibition, we determined the stimulus-response relationships for both excitation and inhibition in individual neurons (Fig. 4M). Light intensity was logarithmically scaled until the peak amplitudes of both responses were consistently maximal. From the resultant stimulus-response curves, we see that excitatory and inhibitory responses scaled proportionally, and that both saturated at similar light intensities.

AON excitation of piriform neurons produces relatively weak disynaptic inhibition, which differentiates it from piriform-topiriform recurrent collateral excitation, and which is invariably coupled to large, fast inhibition. Because these two sets of results were obtained with identical methods of dual viral vectors for channelrhodopsin expression, we compared the relative inhibition to excitation ratio in $\mathrm{AON}$ and piriform (Fig. $4 N$ ). The inhibitory and excitatory conductances were calculated for lightevoked AON-to-piriform and piriform-to-piriform responses (Fig. 4O), with obvious bias of piriform responses toward large inhibition. Even the largest AON-to-piriform excitatory conductance responses $(>10 \mathrm{nS}$ ) failed to evoke strong levels of inhibitory conductance responses comparable to those elicited by piriform activation. This difference is reflected in an inhibitionto-excitation ratio of $3.74 \pm 0.47$ for piriform-to-piriform responses compared with $0.89 \pm 0.20$ for AON-to-piriform responses (Fig. 4P; $p<0.001$ ) (Franks et al., 2011).

This suggests that, even with maximal excitation, there was substantially less recruitment of inhibition than observed for the 

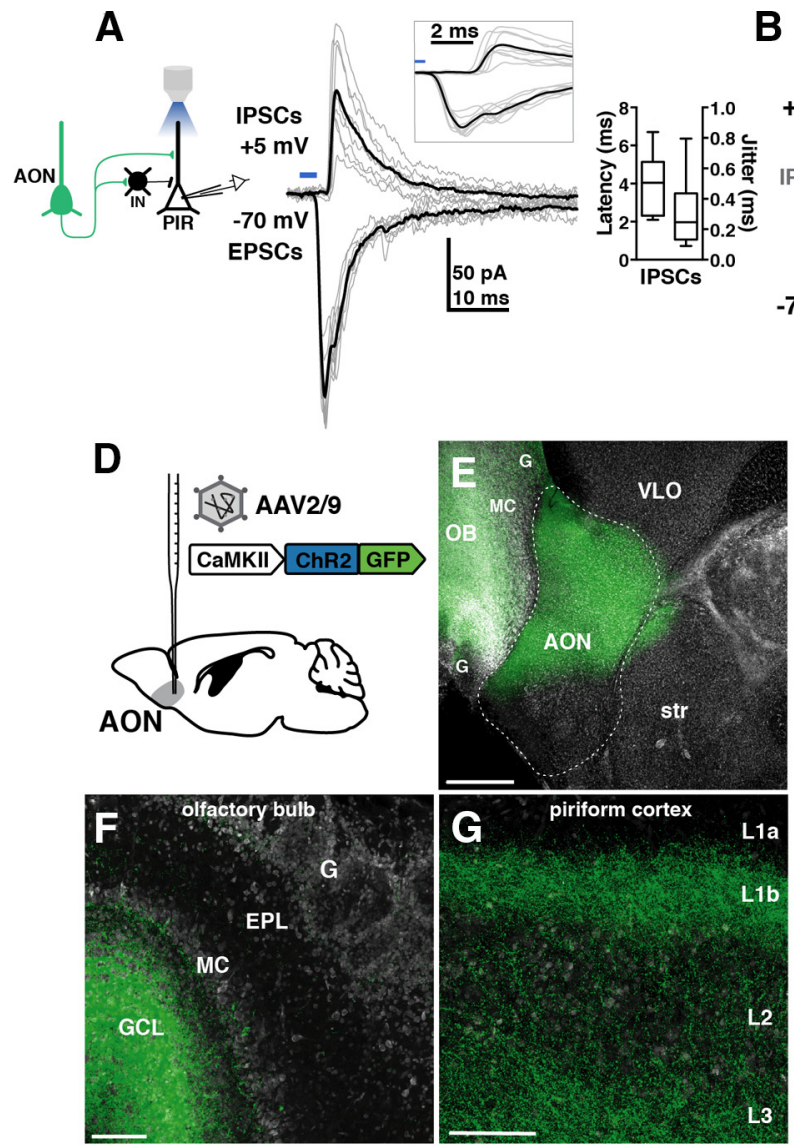

M

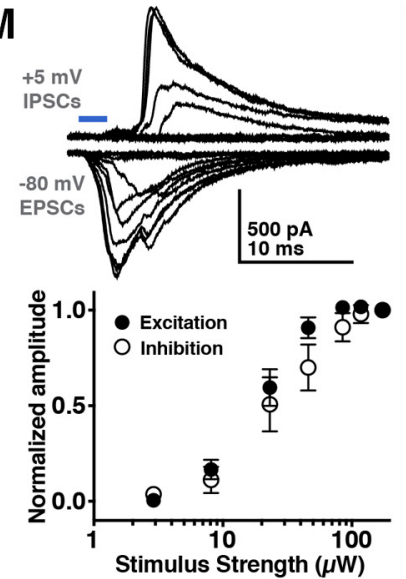

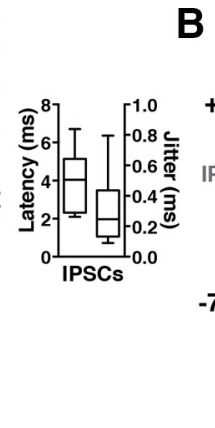

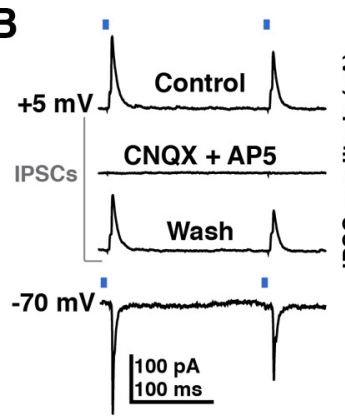

$\mathbf{N}$

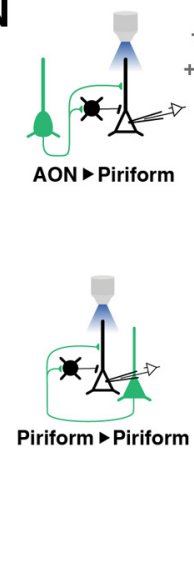

C


H
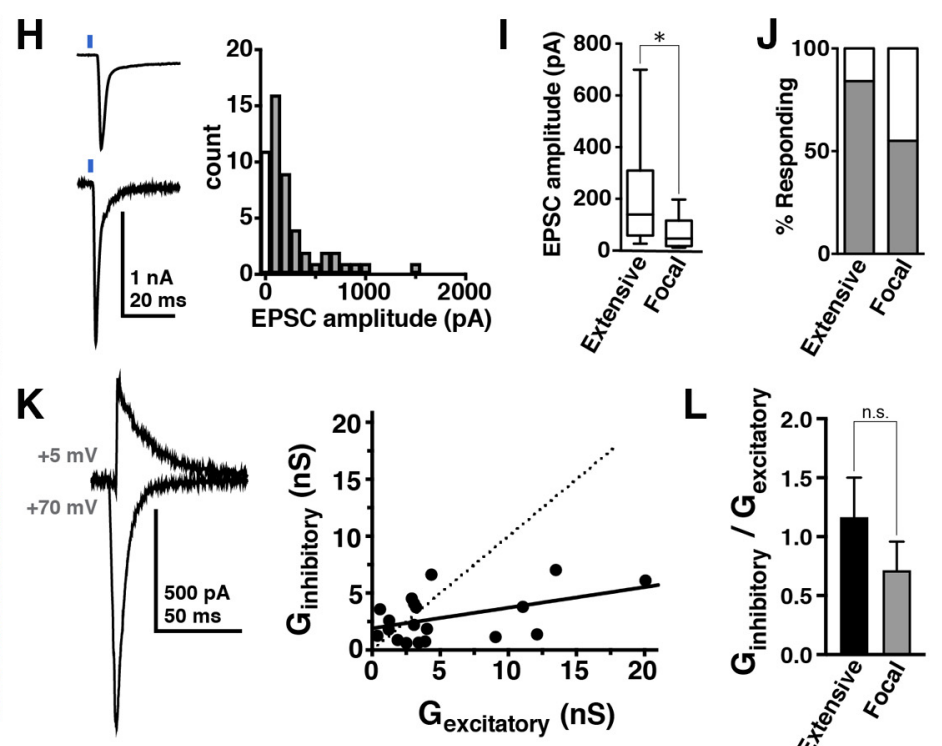

0


$\mathbf{P}$
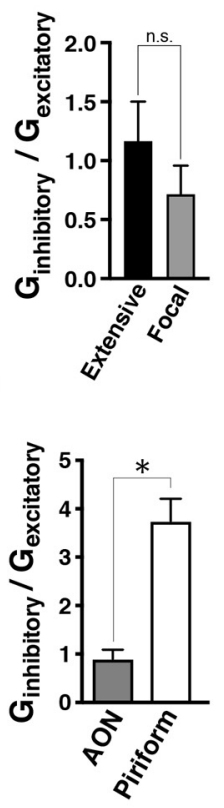

Figure 4. AON inputs activate weak disynaptic inhibition onto piriform neurons. $\boldsymbol{A}$, Voltage-clamp recordings of piriform neurons at $-70 \mathrm{mV}$ (bottom) and $5 \mathrm{mV}$ (top) in response to optical stimulation of AON afferents: overlay of 8 trials at each holding voltage. Black traces represent mean responses. Inset, Magnified timescale of the responses at -70 and $5 \mathrm{mV}$. Right, Plot of latency and jitter for responses recorded at $5 \mathrm{mV}$. For each, median (middle line), 25-75 percentiles (box edges), and 10-90 percentiles are shown (whiskers). Median latency: $4.0 \mathrm{~ms}$ (range 2.1$7.3 \mathrm{~ms}$ ); median jitter: $0.25 \mathrm{~ms}$ (range $0.07-0.89 \mathrm{~ms}$ ). $\boldsymbol{B}$, Response to pair of light pulses under control conditions at $5 \mathrm{mV}$, and responses at $5 \mathrm{mV}$ in the presence of pharmacologic blockers of AMPA- and NMDA-mediated excitatory synaptic transmission, CNQX and AP5, respectively. Bottom, Corresponding excitatory responses in control conditions at - 70 mV. CNQX and AP5 reversibly abolish inhibitory responses, indicating their disynaptic nature (IPSC amplitudes: control 201.8 \pm 53.2 , CNQX + AP5 18.6 \pm 9.0 ; mean \pm SEM, $n=4$ ). $* p<0.001$ (paired $t$ test). C, Left, Maximal EPSCS and IPSCS in an example cell. Right, Total inhibitory conductance ( $G_{\text {inhibitory }}$ ) versus excitatory conductance ( $G_{\text {excitatory }}$ ) plotted for each neuron (slope of linear fit is $0.16 \pm 0.07$, $R^{2}=0.15, n=29$; dotted line of equality provided for reference). Right, ratio of inhibitory to excitatory conductance $\left(G_{\text {inhibitory }} / G_{\text {excitatory }}, 0.71 \pm 0.24\right.$, mean $\left.\pm S E M, n=29\right)$. $D$, Left, Strategy for extensive expression of ChR2 in AON neurons. Animals were injected with nonconditional AAV9 CaMKII-driven ChR2-GFP. CaMKII is a strong promoter that limits expression to glutamatergic neurons. $\boldsymbol{E}$, Parasagittal section through AON injected with AAV9-CaMKII-ChR2-GFP vector. ChR2 is expressed throughout a greater extent of A0N than for the focal method. Scale bar, 500 mm. Dotted line indicates approximate border of AON. OB, Olfactory bulb; G, glomerular layer; MC, mitral cell layer; VLO, ventral/lateral OFC; str, striatum. $F$, Section through the olfactory bulb adjacent to injected AON. The AAV9 serotype has limited/no tropism for olfactory bulb projection neurons. Only centrifugal fibers within the granule cell layer are visible: no YFP ${ }^{+}$mitral or tufted cells were observed. Scale bar, $100 \mu \mathrm{m}$. OB, Olfactory bulb; G, glomerular layer; EPL, external plexiform layer; MC, mitral cell layer; GCL, granule cell layer. G, Pattern of AON axons in piriform cortex in animal injected in AON with AAV9-expressing ChR2 (scale bar, $100 \mu \mathrm{m}$ ), which is similar to the pattern observed with ChR2-YFP in Figure 1D. $\boldsymbol{H}$, Extensive infection and ChR2 expression in the AON leads to larger recorded light-evoked EPSCs in piriform neurons. Left, Two examples of large (>500 pA) light-evoked EPSCs in piriform neurons. Right, Histogram of maximal EPSCS recorded in piriform neurons after extensive AON infection. $I$, Right, Box-and-whisker plot indicating median response amplitude (middle), 25 -75 percentiles (box edges), and 10-90 percentiles (whiskers). Median EPSC with extensive expression 139.9pA (range 4.5-1505.8pA) versus focal expression 47.1 pA (range 4.51-786 pA). $* p<0.0001$ (Mann-Whitney $U$ test). J, Fraction of synaptic responses observed in piriform neurons with extensive (84\%) versus focal (55\%) expression of ChR2 in AON. $\boldsymbol{K}$, Recording of EPSCs (-70 mV) and IPSCs (5 mV) in piriform 
piriform recurrent network. Disynaptic inhibition coupled to piriform recurrent excitation is thought to originate from layer 3 fast-spiking interneurons (Stokes and Isaacson, 2010). AON afferents, on the other hand, likely couple to a unique locus of inhibition or unique interneuron subtypes.

\section{Functional activation of piriform neurons by AON inputs}

We next examined the AON's potential to drive piriform neurons to fire action potentials by performing current-clamp recordings of piriform pyramidal neurons in response to AON photostimulation. All current-clamp experiments were performed in animals with extensive ChR2 expression in the AON to ensure strong excitation. Trains of 5 light pulses at $40 \mathrm{~Hz}$ produced reliable summation of depolarizing EPSPs in piriform neurons (Fig. 5A). The frequency of stimulation throughout our experiments ranged from 20 to $50 \mathrm{~Hz}$, similar to the spike frequencies of AON neurons recorded in response to odors in anesthetized animals (Lei et al., 2006). Temporal summation resulted in nearly a doubling of the depolarization from the beginning to end of the train (first EPSP peak amplitude $=0.98 \pm 0.64 \mathrm{mV}$; fifth EPSP peak amplitude $=$ $1.95 \pm 1.0 \mathrm{mV}$; mean $\pm \mathrm{SEM}, n=9$ neurons). The effect of this summation was particularly notable in some piriform neurons in which the train of AON inputs was sufficient to drive action potential firing (Fig. 5B). Action potentials were more frequently evoked late in the train, indicating that temporal integration was required to reach threshold (Fig. $5 B$, right).

This strong temporal summation of $\mathrm{AON}$ inputs to piriform pyramidal neurons was in marked contrast to the response to similar trains of optogenetic stimuli of piriform recurrent collaterals, in which strong disynaptic inhibition suppressed temporal summation of the piriform EPSP (Franks et al., 2011). Moreover, whereas the piriform recurrent EPSPs are truncated by shortlatency, large-amplitude inhibition, AON EPSPs decay more slowly to baseline at the end of the train, reflecting the modest contribution of inhibition.

We directly examined the differential role of inhibition in temporal integration by delivering stimuli trains in the presence of a $\mathrm{GABA}_{\mathrm{A}}$ receptor antagonist. We recorded trains of light-

$\leftarrow$

neurons in response to AON stimulation. Recording from piriform neuron showing significant excitation, but modest inhibition. Excitatory $(-70 \mathrm{mV})$ and disynaptic inhibitory $(5 \mathrm{mV})$ responses in each cell are plotted. Graph represents peak conductance (solid line, slope $0.21 \pm 0.14, R^{2}=0.27$, line of equality [dotted line] for reference). $L$, Ratio of inhibitory conductance $\left(G_{\text {inhibitory }}\right)$ to excitatory conductance $\left(G_{\text {excitatory }}\right)$ with enhanced activation of AON afferents to piriform $(1.17 \pm 0.33$, mean \pm SEM, $n=19)$ was not significantly greater than for the focal method ( $0.71 \pm 0.24$, mean $\pm \mathrm{SEM}, n=29 ; p=0.27$, unpaired $t$ test) n.S., not significant. $\boldsymbol{M}$, EPSCS ( $-70 \mathrm{mV}$ ) and IPSCS (5 or $10 \mathrm{mV}$ ) were evoked with increasing light intensities in single neurons (2.9-173 $\mu \mathrm{W}$ ). Right, Excitatory (open circles) and inhibitory (closed circles) PSC amplitudes plotted against light stimulus intensity, normalized to the value measured at maximum light intensity. $\mathbf{N}$, Comparison of disynaptic inhibition evoked by optogenetic excitation of AON afferents to disynaptic inhibition evoked by optogenetic activation of piriform recurrent collaterals under similar experimental conditions (Franks et al., 2011). Traces represent the inhibitory current (positive trace) evoked with excitatory current (negative trace) at either AON-to-piriform afferents (top) or piriform-to-piriform afferents (bottom). $\mathbf{0}$, Plot of inhibitory conductance versus excitatory conductance for each recorded neuron for AON-to-piriform (gray circles) or piriform-to-piriform synapses (white circles). Responses measured after extensive ChR2 expression in the AON were included (LVAAV1 and AAV9 data pooled). Inset, Smaller data points for easier visualization. Piriform slope of linear fit 2.45 $\pm 0.21, R^{2}=0.30, n=63$; AON slope $0.25 \pm 0.05, R^{2}=0.31$, $n=48$ ). $P$, Comparison of ratios of inhibitory conductance to excitatory conductance for AON (gray bar, $0.89 \pm 0.20$, mean \pm SEM, $n=48$ ) and piriform (white bar, $3.74 \pm 0.47$, mean $\pm \mathrm{SEM}, n=22$ ). Piriform-to-piriform connections activate significantly larger disynaptic inhibition than AON-to-piriform afferents. $* p<0.0001$ (unpaired $t$ test). evoked AON EPSPs, and for comparison, also recorded electrically evoked "associational" or intracortical EPSPs with a stimulating electrode in layer $1 \mathrm{~b}$ (as in the diagram in Fig. $5 A$ ). Electrical stimulation in layer $1 \mathrm{~b}$ in this manner is known to trigger significant inhibition and should approximate the piriform recurrent collaterals (Ketchum and Haberly, 1993; Franks et al., 2011, their Supplementary Fig. 4). We thus refer to the responses evoked by electrical stimulation in layer $1 \mathrm{~b}$ as piriform recurrent collateral responses. Blockade of inhibition permits temporal summation of recurrent collateral EPSPs but results in little change of summation of AON-evoked EPSPs (Fig. 5C,D). We therefore conclude that differential levels of inhibition do indeed contribute to the differences in extent of temporal integration of the two classes of inputs.

As a further test of the effect of inhibition, we measured temporal summation for the AON-evoked EPSP at different initial holding potentials (Fig. $5 E$ ). The theoretical reversal potential for $\mathrm{Cl}^{-}$in our preparation is close to $-80 \mathrm{mV}$. Therefore, holding the neuron at $-80 \mathrm{mV}$ should reduce the $\mathrm{Cl}^{-}$driving force, and minimize any effects of inhibition, while holding at $-60 \mathrm{mV}$, where there is a large hyperpolarizing $\mathrm{Cl}^{-}$current, should enhance the effects of inhibition. Surprisingly, temporal summation was greatest at $-60 \mathrm{mV}$ and diminished at $-80 \mathrm{mV}$, the opposite of what is expected if temporal integration were primarily regulated by inhibition.

This voltage dependence was further assessed for single EPSPs in both pathways. Consistent with the effect of depolarization to enhance temporal summation of the AON EPSP, the decay of the AON EPSP (indicated by half-width duration) was slowest at $-60 \mathrm{mV}$ and became faster at more negative voltages (Fig. 5F, top). Furthermore, the AON EPSP amplitude did not appreciably decrease with depolarization, despite the decrease in driving force on the excitatory glutamatergic current and increase in driving force on the inhibitory GABAergic current (Fig. $5 F$, bottom). In contrast to the AON EPSP, the piriform EPSP decayed more quickly with depolarization (decreased halfwidth) and the EPSP amplitude became smaller, as expected for the changes in excitatory and inhibitory driving forces.

\section{NMDARs facilitate temporal summation of AON inputs}

The prolongation in deactivation kinetics of the AON EPSP with depolarization suggests a voltage-dependent biophysical mechanism in the pyramidal neuron dendrites. We focused on NMDARs because of their voltage-dependent properties, ability to generate dendritic action potentials in piriform neuron dendrites (Kumar et al., 2018), and strong expression at AON inputs (see Fig. 2D). Blockade of NMDARs with AP5 decreased the amplitude of single AON EPSPs at $-60 \mathrm{mV}$ (Fig. 6A) and caused a small but consistent reduction in the decay time constant and area under the EPSP measured for single stimuli (Fig. 6B). The role of NMDARs was dramatically evident in the effect of AP5 to significantly decrease temporal summation in a reversible manner during a $40 \mathrm{~Hz}$ train of photoactivation of AON inputs (Fig. $6 C)$. This was quantified by the effect of AP5 to decrease the ratio of the EPSP amplitude of the fifth response in the train to the amplitude of the first pulse (control ratio: 1.74 \pm 0.05 ; AP5 ratio: $1.50 \pm 0.07$; mean $\pm \mathrm{SD}, p=0.012, n=4$, paired $t$ test). Therefore, NMDARs prolong the time course of EPSPs at AONto-piriform synapses, allowing for significant synaptic temporal integration of AON inputs at relative depolarization. Combined with the limited activation of disynaptic inhibition, AON synapses have the potential to significantly depolarize and activate piriform principal neurons, especially during brief, physiologically relevant trains of synaptic input. 
A

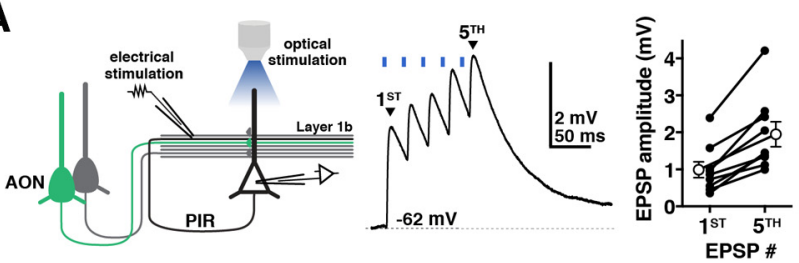

B


E


$\mathbf{F}$
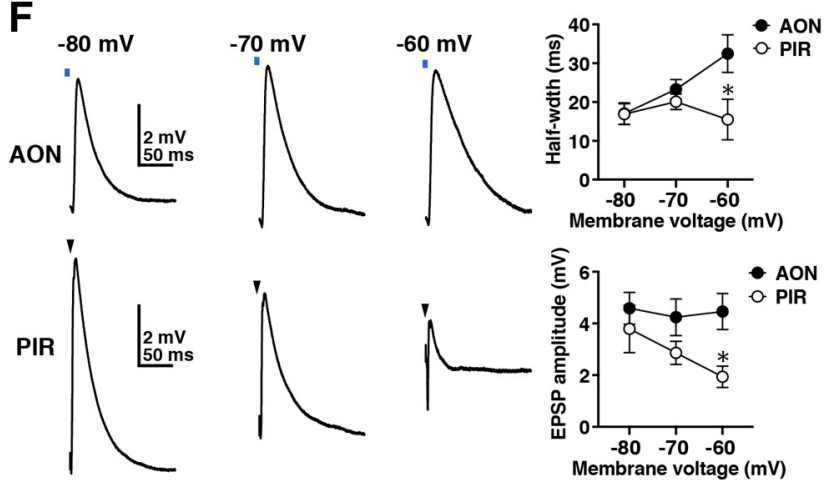

Figure 5. Functional significance of weak disynaptic inhibition. $A$, Left, Diagram of experiment to assess the activating potential of AON synapses onto piriform neurons. AON synapses are activated with optical stimulation. For comparison, the collective associative inputs to piriform in the same neuron, "associational" inputs, were evoked by electrical stimulation in layer $1 \mathrm{~b}$. Based on their properties, they are suspected to be largely represented by piriform recurrent collaterals, and are therefore labeled as piriform inputs (PIR). Middle, Current-clamp recordings of piriform pyramidal neurons in response to AON stimulation with a train of 5 light pulses $(5 \times 2 \mathrm{~ms})$ at $40 \mathrm{~Hz}$. Right, Peak voltage amplitudes were measured for 5-pulse trains in 9 neurons (first EPSP peak $0.98 \pm 0.64 \mathrm{mV}$; fifth EPSP peak: $1.95 \pm 1.0 \mathrm{mV}$; mean \pm SD; $n=9)$. $B$, AON EPSP summation can drive piriform neurons to fire action potentials. Left, Overlaid trials in which light trains $(5 \times 2 \mathrm{~ms}, 40 \mathrm{~Hz})$ drove a piriform neuron to fire (APs in 9 of 20 trials). Middle, Raster plot of action potentials in this neuron. Right, Mean action potential probability within $25 \mathrm{~ms}$ time blocks within a $40 \mathrm{~Hz}$ stimulus train, showing

\section{Discussion}

Our results demonstrate that the AON sends strong glutamatergic excitatory inputs to the piriform cortex, providing a potentially important influence over the cortical odor response. These afferents terminate within the anterior piriform with minimal projections to the posterior piriform cortex. AON afferents densely innervate the proximal apical dendrites of piriform pyramidal neurons, consistent with the anatomic distribution of $\mathrm{YFP}^{+}$fluorescent fibers observed in layer $1 \mathrm{~b}$ of piriform in this study and prior anatomic studies (Haberly and Price, 1978b; Luskin and Price, 1983). Considered together with the known proximal apical bias of piriform recurrent collaterals, the proximal apical dendritic field is a rich area of associative inputs and may serve as a site where piriform recurrent collaterals and $\mathrm{AON}$ afferents interact in functionally meaningful ways. Of note, there was minimal excitation of layer 2 piriform pyramidal neurons in the basal dendritic field, in contrast to the dense AON afferents present in piriform layer 3. This suggests other possible target neurons for these fibers, such as deep pyramidal neurons, or layer 3 interneurons that are distinct from the fast-spiking, parvalbumin-positive/calbindin-positive neurons, and which may include the somatostatin-positive interneurons (e.g., Suzuki and Bekkers, 2010a,b).

A major difference between $\mathrm{AON}$ projections to piriform and the recurrent collaterals within the piriform is the degree of disynaptic inhibition that these two sets of inputs recruit (Fig. 4). Piriform recurrent excitation recruits, and is dominated by, strong disynaptic inhibition, which is likely mediated by perisomatic GABAergic synapses formed by fast-spiking, parvalbumin-positive interneurons in layers $2 / 3$ (Stokes and Isaacson, 2010; Suzuki and Bekkers, 2010a). In contrast, AON excitation

\section{$\leftarrow$}

increased likelihood of firing late in the train. Error bars indicate SEM. Stimulus strength (light intensity) adjusted such that firing only occurred in 50\% of trials for each of the 6 neurons. C, Blockade of inhibition with SR95531 enhances piriform-to-piriform EPSP summation and depolarization but has limited effect on AON EPSPs. Left, Response of piriform neuron to photoactivation of AON afferents by $40 \mathrm{~Hz}$ stimulus train in the presence (purple trace) and absence (black trace) of the GABA(A)-receptor antagonist SR95531. Middle, EPSP amplitudes at each pulse in the train for 6 neurons. Right, Quantification of summation as ratio of fifth EPSP amplitude in train to first EPSP in train in presence and absence of SR95531 (control: $1.78 \pm 0.24$; SR95531: $1.56 \pm 0.24$; mean \pm SEM, $n=6, p=0.15$, n.s., not significant paired $t$ test). $\boldsymbol{D}$, Train of EPSPs evoked by electrical stimulation of associational (piriform) inputs in presence and absence of $\mathrm{GABA}_{\mathrm{A}}$ blocker. Middle, There is marked enhancement of the piriform-to-piriform response with blockade of inhibition. Right, Ratio of EPSP amplitude shows trend toward increased summation at piriform-to-piriform summation with inhibition blocked (control: $1.78 \pm 0.28$; SR95531: $2.47 \pm 0.45$; mean \pm SEM; $n=5, p=0.32$, n.s., not significant paired $t$ test). $\boldsymbol{E}$, Left, Trains of EPSPs evoked by photoactivation of AON afferents $(5 \times 2 \mathrm{~ms}, 50 \mathrm{~Hz})$, while adjusting the pyramidal neuron membrane potential to -60 , -70 , and $-80 \mathrm{mV}$ with small current injections. Note increased summation and depolarization at $-60 \mathrm{mV}$. Right, EPSP amplitude, normalized to the amplitude of the first response in the train, showing enhanced summation of EPSPs at $-60 \mathrm{mV}$ compared to $-80 \mathrm{mV}$. Normalized amplitude at EPSP \#5 is $1.20 \pm 0.05$ at $-60 \mathrm{mV}$ versus $0.84 \pm 0.04$ at $-80 \mathrm{mV}$ (mean $\pm \mathrm{SEM}, n=5$ ). $* p=0.0008$ ( $t$ test). Error bars at $-70 \mathrm{mV}$ were omitted for easier visualization. $\boldsymbol{F}$, Single EPSPs at varying potentials. Top left, Single light pulses to evoke single AON EPSPs at different subthreshold voltages. Bottom left, Single pulses of electrical stimulation to evoke piriform recurrent collaterals. Top right, Quantification of the time course of single EPSPs evoked at different membrane potentials. The time course for each pathway was measured as the EPSP half-width duration. Piriform (open circles) EPSP decay is attenuated at depolarized potentials, but AON responses (closed circles) are not attenuated, but prolonged. At $-60 \mathrm{mV}$, half-width for piriform is $15.5 \pm 5.2 \mathrm{~ms}$, whereas that for AON is $32.5 \pm 4.8 \mathrm{~ms}$ (mean $\pm \mathrm{SEM}, n=5$ ). $* p=0.044$ ( $t$ test). Bottom right, Peak amplitudes of single EPSPs for each pathway at the corresponding starting voltages (mean \pm SEM, $n=5) . * p=0.014, t$ test. 



Figure 6. Enhancement of AON EPSP summation with depolarization is NMDAR-dependent. $A, A O N$ EPSPs are sensitive to blockade of NMDARs. Left, Single AON EPSP in the absence (black trace) and presence (blue trace) of AP5, a selective NMDAR antagonist, recorded at $-60 \mathrm{mV}$. Right, EPSP amplitudes in the presence and absence of AP5 for 5 neurons (control: $4.6 \pm 0.18 \mathrm{mV}$; AP5: $3.4 \pm 0.48 \mathrm{mV}$, mean \pm SEM, $n=5 ; * p=0.004$, paired $t$ test). $\boldsymbol{B}$, EPSP time course is sensitive to NMDAR blockade. Left, Time constant of EPSP decay $\left(\tau_{\text {off }}\right)$ determined from monoexponential best fit (control: $23.3 \pm 3.63 \mathrm{~ms}$; AP5: $17.2 \pm 4.15 \mathrm{~ms}$, $n=5, * p=0.005$ ). Right, Area under the curve of EPSPs (control: $0.17 \pm 0.05 \mathrm{mV} \cdot \mathrm{s}, \mathrm{AP5}$ : $0.10 \pm 0.03 \mathrm{mV} \cdot \mathrm{s}$, mean $\pm \mathrm{SEM}, \mathrm{n}=5 ; * p=0.008$, paired $t$ test). $C$, Effect of NMDAR blockade on temporal summation of AON EPSPs. Left, Train of light pulses $(5 \times 2 \mathrm{~ms}, 40 \mathrm{~Hz})$ under control (black trace) conditions and with AP5 (blue trace). Summation returns with wash of AP5 from the bath (gray trace). Right, Average effects of AP5 on temporal integration, expressed as ratio of amplitude fifth EPSP peak to amplitude of first EPSP peak (control; $1.74 \pm 0.05$ vs AP5: $1.50 \pm 0.07$; mean $\pm S D, n=4 ; * p=0.012$, paired $t$ test).

within piriform evokes only weak disynaptic inhibition, which does not constrain excitation, even with increasing intensity of excitatory drive or increasing numbers of activated AON afferents. This suggests that AON afferents to piriform cortex, while providing weaker excitation than recurrent collaterals, are less constrained by inhibition. Moreover, it indicates that AON afferents likely interact with a unique interneuron subtype within piriform cortex that mediates this weaker disynaptic inhibition, and may be a target for neuromodulation.

We also found that synapses from AON afferents to the piriform cortex have high levels of NMDARs, whose voltage-dependent properties help amplify synaptic excitation to patterns of input that lead to significant postsynaptic depolarization (Kumar et al., 2018). Because AON excitatory synapses are less subject to feedforward inhibition (as for piriform collaterals), synaptic depolarization during a brief train of AON synaptic input enhances the voltage-dependent activation of local NMDARs, resulting in further depolarization, prolongation of EPSPs, and enhanced temporal integration. In some neurons, this temporal summation is sufficient to induce firing. This unrestrained NMDAR activation has several important potential consequences: (1) the AON may directly evoke action potential firing in some piriform neurons; (2) it may directly integrate with LOT inputs without the strict timing dependence of piriform-LOT integration; (3) AON synapses may more easily undergo NMDAR-dependent LTP; and (4) given that they have similar synaptic loci, the AON may provide the depolarization or $\mathrm{Ca}^{2+}$ influx to facilitate plasticity at coincidentally active piriform recurrent synapses (or other associative synapses at the apical dendrite). Thus, AON-to-piriform afferents have numerous potential mechanisms through which the AON may enhance or shape the piriform response to odor.

We made considerable efforts to confirm that our focal viral injection methods produced ChR2 expression that was restricted to the AON. Because of the proximity of the orbitofrontal areas and the known anatomic projections from orbitofrontal cortex (OFC) to piriform cortex (Illig, 2005), we cannot exclude potential effects of OFC afferents to piriform, but the robust and consistent expression of ChR2 in the AON, verified by restricted fluorescence and measured photocurrents, makes this unlikely. Nevertheless, the OFC is a potentially important contributor to the associative piriform cortex network, and these projections merit dedicated future study.

Detailed anatomic studies have shown that the $\mathrm{AON}$ is not homogeneous but has important regional differences in cytoarchitecture, gene expression, and connectivity (Haberly, 2001; Meyer et al., 2006). For instance, a major distinction is made between the dorsal, lateral, and ventroposterior portions of the $\mathrm{AON}$ and its medial subdivision based on observation of distinct neuronal morphology and that the former areas receive the majority of bulbar afferents via the LOT (Haberly and Price, 1978b). We did not systematically investigate regional differences in the AON projections to piriform cortex, and ChR2 expression was not restricted to singular subdivisions but was expressed and activated as a collective projection from par principalis to piriform (pars externa was not targeted). Future systematic examination of the properties of afferents to piriform from distinct subdivisions of $\mathrm{AON}$, and the contralateral $\mathrm{AON}$, including complementary retrograde labeling of $\mathrm{AON}$ neurons projecting to piriform, will be necessary for a precise understanding of the regional organization of $\mathrm{AON}$-piriform connectivity.

The role of the AON in olfactory signaling remains enigmatic, but it is becoming clearer that it occupies a central position within the olfactory hierarchy. The AON receives early convergent input from the olfactory bulb and sends reciprocal connections back to the bulb. It also projects bilaterally to nearly all olfactory areas. These centrifugal bulbar projections contact numerous bulbar interneurons, which lead to a net inhibitory influence of the AON onto the olfactory bulb, and may provide a mechanism for selectively increasing the signal-to-noise of the bulbar response to behaviorally meaningful odors in a state-dependent manner. Our results provide evidence that AON afferents to piriform cortex, contrary to centrifugal afferents to the olfactory bulb, will have a net excitatory effect on higher-order associational processing of olfactory information (Boyd et al., 2012; Markopoulos et al., 2012). This is also distinct from the dense excitatory recurrent connections from piriform neurons, whose net effect is inhibitory, both in slice (Franks et al., 2011) and in vivo (Bolding and Franks, 2018).

The AON may play an important behavioral role in olfactory processing based on studies of the action of the social neuropeptide oxytocin. Induced oxytocin release in the AON enhances socially motivated behaviors, and oxytocin both increases excitability of AON neurons and facilitates the inhibitory effects of feedback projections from the AON to the olfactory bulb (Oettl et al., 2016). This raises the question as to how such 
neuromodulation alters the synaptic effects of $\mathrm{AON}$ projections to piriform cortex and the associative circuitry within the piriform. Further evidence implicating the AON in odor information processing is provided by the finding that activation of the AON (pars medialis) through projections from the ventral hippocampus results in impaired performance on olfactory-dependent behaviors (Aqrabawi et al., 2016).

Our results provide strong motivation for systems-level studies into the functional roles in olfaction of the $\mathrm{AON} /$ cortex and its projections to the piriform cortex. A critical future experiment will be to functionally activate and inhibit the AON afferents to piriform cortex in the behaving animal, with experiments carefully designed to dissect the contributions of AON projections to piriform compared with AON projections to other olfactory areas and centrifugal projections to the olfactory bulb. Such studies will eventually lead to a deeper understanding as to how the AON modifies and shapes the piriform odor response, how this is regulated by neuromodulatory inputs, and how the AON contributes to olfactory learning and behavior.

\section{References}

Aqrabawi AJ, Browne CJ, Dargaei Z, Garand D, Khademullah CS, Woodin MA, Kim JC (2016) Top-down modulation of olfactory-guided behaviours by the anterior olfactory nucleus pars medialis and ventral hippocampus. Nat Commun 7:13721.

Bolding KA, Franks KM (2018) Recurrent cortical circuits implement concentration-invariant odor coding. Science 361:eaat6904.

Boyd AM, Sturgill JF, Poo C, Isaacson JS (2012) Cortical feedback control of olfactory bulb circuits. Neuron 76:1161-1174.

Boyden ES, Zhang F, Bamberg E, Nagel G, Deisseroth K (2005) Millisecondtimescale, genetically targeted optical control of neural activity. Nat Neurosci 8:1263-1268.

Brunjes PC, Kay RB, Arrivillaga JP (2011) The mouse olfactory peduncle. J Comp Neurol 519:2870-2886.

Cetin A, Komai S, Eliava M, Seeburg PH, Osten P (2006) Stereotaxic gene delivery in the rodent brain. Nat Protoc 1:3166-3173.

Choi GB, Stettler DD, Kallman BR, Bhaskar ST, Fleischmann A, Axel R (2011) Driving opposing behaviors with ensembles of piriform neurons. Cell 146:1004-1015.

Datiche F, Litaudon P, Cattarelli M (1996) Intrinsic association fiber system of the piriform cortex: a quantitative study based on a cholera toxin B subunit tracing in the rat. J Comp Neurol 376:265-277.

Davison IG, Ehlers MD (2011) Neural circuit mechanisms for pattern detection and feature combination in olfactory cortex. Neuron 70:82-94.

Franks KM, Isaacson JS (2005) Synapse-specific downregulation of NMDA receptors by early experience: A critical period for plasticity of sensory input to olfactory cortex. Neuron 47:101-114.

Franks KM, Isaacson JS (2006) Strong single-fiber sensory inputs to olfactory cortex: implications for olfactory coding. Neuron 49:357-363.

Franks KM, Russo MJ, Sosulski DL, Mulligan AA, Siegelbaum SA, Axel R (2011) Recurrent circuitry dynamically shapes the activation of piriform cortex. Neuron 72:49-56.

Haberly LB (2001) Parallel-distributed processing in olfactory cortex: New insights from morphological and physiological analysis of neuronal circuitry. Chem Senses 26:551-576.

Haberly LB, Price JL (1978a) Association and commissural fiber systems of the olfactory cortex of the rat: I. Systems originating in the piriform cortex and adjacent areas. J Comp Neurol 178:711-740.

Haberly LB, Price JL (1978b) Association and commissural fiber systems of the olfactory cortex of the rat: II. Systems originating in the olfactory peduncle. J Comp Neurol 181:781-807.

Hagiwara A, Pal SK, Sato TF, Wienisch M, Murthy VN (2012) Optophysiological analysis of associational circuits in the olfactory cortex. Front Neural Circuits 6:18.

Hegemann P, Ehlenbeck S, Gradmann D (2005) Multiple photocycles of channelrhodopsin. Biophys J 89:3911-3918.
Illig KR (2005) Projections from orbitofrontal cortex to anterior piriform cortex in the rat suggest a role in olfactory information processing. J Comp Neurol 488:224-231.

Illig KR, Eudy JD (2009) Contralateral projections of the rat anterior olfactory nucleus. J Comp Neurol 512:115-123.

Johnson DM, Illig KR, Behan M, Haberly LB (2000) New features of connectivity in piriform cortex visualized by intracellular injection of pyramidal cells suggest that "primary" olfactory cortex functions like "association" cortex in other sensory systems. J Neurosci 20:6974-6982.

Kanter ED, Haberly LB (1990) NMDA-dependent induction of long-term potentiation in afferent and association fiber systems of piriform cortex in vitro. Brain Res 525:175-179.

Ketchum KL, Haberly LB (1993) Membrane currents evoked by afferent fiber stimulation in rat piriform cortex. I. Current source-density analysis. J Neurophysiol 69:248-260.

Kumar A, Schiff O, Barkai E, Mel BW, Poleg-Polsky A, Schiller J (2018) NMDA spikes mediate amplification of inputs in the rat piriform cortex. Elife 7:e38446.

Le Y, Miller JL, Sauer B (1999) GFPcre fusion vectors with enhanced expression. Anal Biochem 270:334-336.

Lei H, Mooney R, Katz L (2006) Synaptic integration of olfactory information in mouse anterior olfactory nucleus. J Neurosci 26:12023-12032.

Luna VM, Schoppa NE (2008) GABAergic circuits control input-spike coupling in the piriform cortex. J Neurosci 28:8851-8859.

Luskin MB, Price JL (1983) The topographic organization of associational fibers of the olfactory system in the rat, including centrifugal fibers to the olfactory bulb. J Comp Neurol 216:264-291.

Markopoulos F, Rokni D, Gire DH, Murthy VN (2012) Functional properties of cortical feedback projections to the olfactory bulb. Neuron 76:1175-1188.

Meyer EA, Illig KR, Brunjes PC (2006) Differences in chemo- and cytoarchitectural features within pars principalis of the rat anterior olfactory nucleus suggest functional specialization. J Comp Neurol 498:786-795.

Oettl LL, Ravi N, Schneider M, Scheller MF, Schneider P, Mitre M, da Silva Gouveia M, Froemke RC, Chao MV, Young WS, Meyer-Lindenberg A, Grinevich V, Shusterman R, Kelsch W (2016) Oxytocin enhances social recognition by modulating cortical control of early olfactory processing. Neuron 90:609-621.

Petreanu L, Mao T, Sternson SM, Svoboda K (2009) The subcellular organization of neocortical excitatory connections. Nature 457:1142-1145.

Poo C, Isaacson JS (2009) Odor representations in olfactory cortex: "sparse" coding, global inhibition, and oscillations. Neuron 62:850-861.

Poo C, Isaacson JS (2011) A major role for intracortical circuits in the strength and tuning of odor-evoked excitation in olfactory cortex. Neuron 72:41-48.

Roland B, Deneux T, Franks KM, Bathellier B, Fleischmann A (2017) Odor identity coding by distributed ensembles of neurons in the mouse olfactory cortex. Elife 6:e26337.

Stern M, Bolding KA, Abbott LF, Franks KM (2018) A transformation from temporal to ensemble coding in a model of piriform cortex. Elife 7:e34831.

Stettler DD, Axel R (2009) Representations of odor in the piriform cortex. Neuron 63:854-864.

Stokes CC, Isaacson JS (2010) From dendrite to soma: dynamic routing of inhibition by complementary interneuron microcircuits in olfactory cortex. Neuron 67:452-465.

Suzuki N, Bekkers JM (2006) Neural coding by two classes of principal cells in the mouse piriform cortex. J Neurosci 26:11938-11947.

Suzuki N, Bekkers JM (2010a) Distinctive classes of GABAergic interneurons provide layer-specific phasic inhibition in the anterior piriform cortex. Cereb Cortex 20:2971-2984.

Suzuki N, Bekkers JM (2010b) Inhibitory neurons in the anterior piriform cortex of the mouse: classification using molecular markers. J Comp Neurol 518:1670-1687.

Suzuki N, Bekkers JM (2011) Two layers of synaptic processing by principal neurons in piriform cortex. J Neurosci 31:2156-2166.

Suzuki N, Bekkers JM (2012) Microcircuits mediating feedforward and feedback synaptic inhibition in the piriform cortex. J Neurosci 32:919-931.

Yizhar O, Fenno LE, Davidson TJ, Mogri M, Deisseroth K (2011) Optogenetics in neural systems. Neuron 71:9-34.

Zhang F, Wang LP, Boyden ES, Deisseroth K (2006) Channelrhodopsin-2 and optical control of excitable cells. Nat Methods 3:785-792. 